\title{
Relativistic AGN jets III. Synthesis of synchrotron emission from Double-Double Radio Galaxies
}

\author{
S. Walg, ${ }^{1,2 \star}$ A. Achterberg, ${ }^{1}$ S. Markoff, ${ }^{2}$ R. Keppens, ${ }^{3}$ O. Porth ${ }^{2}$ \\ ${ }^{1}$ Department of Astrophysics/IMAPP, Radboud University, P.O. Box 9010, 6500 GL Nijmegen, The Netherlands. \\ ${ }^{2}$ Astronomical Institute "Anton Pannekoek," University of Amsterdam, Science Park 904, 1098 XH Amsterdam, The Netherlands \\ ${ }^{3}$ Centre for mathematical Plasma Astrophysics, Department of Mathematics, KU Leuven, Celestijnenlaan 200B, 3001 Heverlee, Belgium
}

30 July 2020 (accepted 23 July 2020, DOI: 10.1093/mnras/staa2195)

\begin{abstract}
The class of Double-Double Radio Galaxies (DDRGs) relates to episodic jet outbursts. How various regions and components add to the total intensity in radio images is less well known. In this paper we synthesize synchrotron images for DDRGs based on special relativistic hydrodynamic simulations, making advanced approximations for the magnetic fields. We study the synchrotron images for: Three different radial jet profiles; Ordered, entangled or mixed magnetic fields; Spectral ageing from synchrotron cooling; The contribution from different jet components; The viewing angle and Doppler (de-)boosting; The various epochs of the evolution of the DDRG. To link our results to observational data, we adopt to J1835+6204 as a reference source. In all cases the synthesized synchrotron images show two clear pairs of hotspots, in the inner and outer lobes. The best resemblance is obtained for the piecewise isochoric jet model, for a viewing angle of approximately $\vartheta \sim-71^{\circ}$, i.e. inclined with the lower jet towards the observer, with predominantly entangled ( $~ 70$ per cent of the magnetic pressure) in turbulent, rather than ordered fields. The effects of spectral ageing become significant when the ratio of observation frequencies and cut-off frequency $v_{\mathrm{obs}} / v_{\infty, 0} \gtrsim 10^{-3}$, corresponding to $\sim 3 \cdot 10^{2} \mathrm{MHz}$. For viewing angles $\vartheta \lesssim-30^{\circ}$, a DDRG morphology can no longer be recognized. The second jets must be injected within $\lesssim 4$ per cent of the lifetime of the first jets for a DDRG structure to emerge, which is relevant for Active Galactic Nuclei feedback constraints.
\end{abstract}

Key words: galaxies: jets - hydrodynamics - magnetic fields -radiation mechanisms: nonthermal - relativistic processes -software: simulations

\section{INTRODUCTION}

Active Galactic Nuclei (AGN) jets that are created by the central engine of a powerful radio galaxy are capable of growing up to megaparsec scales (Dabhade et al. 2017). They can be active for tens or hundreds of megayears (Bird et al. 2008; Turner \& Shabala 2015). Some radio galaxies are thought to have gone through an episodic outburst cycle, where the central engine has been turned off for some time. If this time of intermittency is less than the time it takes for a jet plasma element to travel from the central engine to the jet-head, there will be a phase only seen in radio images where two distinct jets and counter jets will be visible, as well as their corresponding hotspots, forming a double-double radio galaxy (DDRG).

The class of double-double radio galaxies has been discovered a relatively short time ago (Schoenmakers et al. 2000|Schoenmakers et al. 2000, Kaiser, Schoenmakers \& Röttgering 2000). It is characterized by having two aligned distinct pairs of radio lobes originating from the same central engine of the AGN. More than 20 DDRGs have been clearly identified so far (see for example Saikia \& Jamrozy 2009 Nandi et al. 2019, Kuźmicz et al. 2017 or Mahatma et al. 2019), but with the increasing sensitivities and radio bandwidth in novel radio telescopes such as LOFAR, the number of detected radio loud AGN or LOFAR DDRGs has increased significantly (see for example the GLEAM survey, Callingham et al. 2017, or the LOFAR Two-metre Sky Survey (LoTSS), see Shimwell et al. 2019. Hardcastle et al. 2019, Mahatma et al.|2019) so that the number of detectable DDRGs might also grow significantly (Orrù et al. 2015). As the synchrotron emission fades rapidly, the higher sensitivity and low frequency coverage as achieved by LOFAR is crucial in that aspect. The typical morphology of these sources, as well as studies on the spectral age of different regions, suggest that DDRGs are the result of episodic jet activity (Mahatma et al. 2019, Schoenmakers et al. 2000, Saikia \& Jamrozy 2009. Marecki et al. 2016, Nandi et al. 2017, 2019). The jet size can be extremely large, while the associated $\sim$ Myr time scales for source 
evolution excludes us to witness the episodic scenario within a single, individual source.

The radio emission observed in DDRGs is mainly (nonthermal) synchrotron radiation. It is produced by relativistic electrons that are spiralling along magnetic field lines which are carried along by the plasma. What we see is the sum (superposition) of all synchrotron-radiating particles. However, for jets with a radial structure and in the case of episodic activity, different jet components might have very different intensities, leading to different brightness variations in synchrotron radiation. Brightness variations in radio images and the frequency at which the source is observed can strongly influence the general observed morphology of these sources. This can be a complicating factor when comparing an observed DDRG to a synthesized map of a numerical simulation. To better understand the processes that lead to the observed DDRGs, a study on the synchrotron radiation coming from various jet components is essential.

In the past few decades, a number of numerical studies on episodic jet activity have been performed (see e.g. Wilson 1984, Clarke \& Burns 1991, Chon et al. 2012, Mendygral, Jones \& Dolag 2012, Refaelovich \& Soker 2012, Stepanovs, Fendt \& Sheikhnezami 2014, Walg et al. 2014, Lee et al. 2016). In Walg et al. (2014), a $2.5 D$ (i.e. accounting for all three flow components but assuming axisymmetric evolution) cylindrical symmetric special relativistic hydrodynamical (SRHD) study was performed on episodic activity of relativistic AGN jets. In that work, the density, pressure and jet velocity were chosen such that they correspond well to those inferred from observations of a typical DDRG (for example Konar \& Hardcastle 2013). The jets discussed in Walg et al. (2013) (hereafter SW1) and Walg et al. (2014) (hereafter SW2) have radial structure, consisting of a high-Lorentz factor jet spine and slower jet sheath. This is inspired from observations, showing evidence for spine-sheath structured jets (see e.g. Laing \& Bridle 2014). The main emphasis in SW2 was on the integrity of this structure as the jet propagates, and how the different jet components mix.

\section{Main focus of this research}

Synchrotron emissivity is determined by the number density of relativistic particles, the frequency of the photons and the strength of the magnetic field component perpendicular to the particle's velocity. Bulk motion of the source material, and in particular motion along the line-of-sight, influences the observed intensity through the Doppler effect. This will be discussed in more detail in section 2 In order to create a synthesized synchrotron map, one requires a full special relativistic magnetohydrodynamic (SRMHD) treatment with additional dynamical tracing of a relativistic particle population. Presently, there are a great number of relativistic magnetohydrodynamics (MHD) codes. A recent study compares a significant number of (general relativistic) magneto-hydrodynamics (GRMHD) codes including Athena++, BHAC, Cosmos++, ECHO, H-AMR, iharm3D, HARM-Noble, IllinoisGRMHD, Koral (Porth et al. 2019), but in this study GR is not needed when studying the larger scales in the jets. Despite the benefits of having detailed data of the evolution of the magnetic fields, SRMHD simulations are computationally much more expensive than pure special relativistic hydrodynamic (SRHD) simulations.

Close to the AGN, magnetic fields are dynamically important and may be linked to the mechanism that collimates the jet flow (e.g. Begelman, Blandford \& Rees 1984, Bromberg et al. 2011 ,
Blandford, Meier \& Readhead 2019, Chatterjee et al. 2019 ). At large $(\mathrm{Mpc})$ scales, when the jet has reached its terminal velocity, the fields are no longer dynamically important as the jet flow is expected to reach strongly supersonic/super-Alfvénic speeds. Moreover when the jets have switched off, the plasma will mostly evolve adiabatically. In that case, we make the often used assumption that there is equipartition between the pressure stored in the magnetic field and the gas pressure from the relativistic particle population. From our SRHD simulation, this is the simplest way to handle the unknown magnetic field strength quantitatively, and equipartition fields are close to the state in which the total energy of the plasma is minimized. We will distinguish between two underlying magnetic field topologies, namely ordered versus turbulent field, which together build up the deduced magnetic pressure. In SRMHD simulations, one would need excessive resolutions to properly account for turbulent magnetic fields. From our grid-adaptive SRHD runs, we get extended turbulent mixing regions as discussed in SW1 and SW2. Although some studies (Croston et al. 2005, 2018, Mahatma et al. 2020) show evidence for field strengths in the lobes that are lower than equipartition estimates, we find it reasonable to assume that the gas pressure from the relativistic particles is directly proportional to the mass fraction of the different jet components. In the numerical simulations in this paper, we have used additional tracers, to identify the various jet components at all times, allowing to determine mass fractions of jet material in different jet episodes or different layers (spine versus sheath). This allows us to infer the magnetic field strength directly from the bulk gas pressure and tracer values, without the need for dynamically evolving the magnetic fields.

The synchrotron emission from AGN jets can usually be represented by a power law in frequency $v$,

$S_{v} \propto v^{-\alpha}$,

over a wide range of frequencies, where $\alpha$ is the spectral index. This is the emission expected from a population of relativistic electrons with an energy distribution $N(\epsilon) \mathrm{d} \epsilon \propto \epsilon^{-(2 \alpha+1)} \mathrm{d} \epsilon$ (e.g. Longair 2011. Ch. 8, or Rybicki \& Lightman 1986, Ch. 6). Here $\epsilon$ is the electron energy. The power-law continues up to some cut-off frequency $v_{\infty}$, where a break in the spectrum occurs either due to synchrotron losses of the relativistic electrons or the fact that the acceleration mechanism has a limiting (maximum) energy.

When a power law distribution is assumed for the population of relativistic particles at the jet inlet, the evolution of this population can be calculated in two ways: i) either it can be inferred from the bulk mass density and the mass fraction of jet material; ii) it can be deduced from advecting a quantity proportional to the cut-off energy $\epsilon_{\infty}$ (see for example Camus 2009. Ch. 4; or Camus et al. 2009). Both methods will be discussed in this paper. We utilize the tracers for various jet components to full extent. This allows us to study the influence of the various jet components on the brightness variations within a synchrotron map. Moreover, we will impose various magnetic field configurations on our numerical simulations and study the influence on the overall morphology. Finally, we utilize the jet tracers to give an estimation of how large-scale ordered fields transform into entangled fields, so a mixed magnetic field configuration can be used. In this work we focus on synthesizing synchrotron maps from the same simulations that were presented in SW2. In SW2, we emphasized that subtly different dynamics and mixing properties arise when jets that differ in their radial stratification interact. This will undoubtedly reflect in synthetic synchrotron views, and this will become evident in this paper. The synchrotron views 
are expected to be sensitive to the deduced magnetic field configurations, an aspect to be investigated here as well. Moreover, radiative losses (synchrotron ageing) can be a decisive factor in the overall radio appearance. Therefore, we will:

- compare the emission from three different jet models: a homogeneous jet; a piecewise isochoric jet; and an isothermal jet;

- compare the emission for various different imposed magnetic field configurations;

- study the effect of dimming due to synchrotron ageing of the first jet on the radio morphology;

- compare the emission coming from the initial jet and the restarted jet separately;

- consider different viewing angles for the DDRG phase, taking into account the effect of the Doppler factor;

- consider the emission from the source at various phases of the outburst cycle.

As a reference DDRG source, we will consider J1835+6204, (observed at $4860.1 \mathrm{MHz}$ with the VLA in the B or D configuration, with beam sizes 1.14 " and 1.71 "), for which various observational results exist in the literature (Konar et al. 2012, 2013). In Konar et al. (2013), the duration of the quiescent phase was estimated to be $\sim 1 \mathrm{Myr}$, as short as 4.5 per cent of the previous active phase, while the age of the source is estimated to be $\sim 22 \mathrm{Myr}$. Estimates of the speed of the jet spine, from a sample of radio galaxies including J1835+6204, were made in Konar \& Hardcastle (2013), inferring Lorentz factors even above 10, to explain the observations. The spectral index for this source is $\alpha \sim 0.818$. Our models for DDRG jets discussed in SW2 adopted parameters inspired from observations, with e.g. the first jet injection lasting for $15.3 \mathrm{Myr}$, a (smoothly varying) switching-off of the central engine between 15.3 and up to $16 \mathrm{Myr}$ (see Eqns. (3)-(4) and Fig. 2 in SW2), and a subsequent jet injection for another 6.8 Myr. The Lorentz factor is adopted as 3.11 for the homogeneous jet, while the spine-sheath models (isochoric or isothermal jets) have a central spine with a Lorentz factor 6 . All three jet models derive parameters from a typical kinetic jet luminosity of a few $\times 10^{46} \mathrm{erg} \mathrm{s}^{-1}$. Details of the jet models are described in SW1, where it was shown how different internal jet structures can lead to dramatic differences in maintaining jet integrity, and on their overall propagation speeds. In SW2, the same jet stratification models were then used to discuss their interaction in a DDRG scenario, and here we follow up with their synthetic radio views.

\section{THEORETICAL BACKGROUND}

We assume that the bulk flow of jet material contains a population of relativistic particles. A recent study suggests that the composition of relativistic jets in FR-II radio galaxies consists of electrons, positrons and protons (Kawakatu, Kino \& Takahara 2016). In our simulations, we do not consider low-power (FR-I class) jets, which may entrain significantly more material while propagating through the host galaxy and the Intergalactic medium (Laing \& Bridle 2014). Such jets are more vulnerable to instabilities at the jetcocoon and cocoon-intergalactic medium interfaces, with resultant turbulent mixing. In our FR-II scenarios, we model all entrainment with the surrounding medium in the simulations, except for any entrainment aspects close to the central engine, which we exclude. We treat synchrotron emission in the optically thin limit. The total intensity of synchrotron radiation, $I(X, Y)$, at sky position $(X, Y)$ and frequency $v$ is completely determined by the synchrotron emissivity coming from the plasma of non-thermal electrons. Hereafter, we shall denote $\boldsymbol{n}$ as a unit vector along the line of sight pointing at the observer, measured in the observers frame. The intensity measured at the sky position $(X, Y)$ is calculated by integrating the emissivity $j_{v}$ along the line of sight $Z \boldsymbol{n}$, perpendicular to the sky coordinate plane. It equals:

$I_{v}=\int \mathrm{d} Z j_{v}(\boldsymbol{r}, t)$,

where $j_{v}(\boldsymbol{r}, t)$ is the synchrotron emissivity at time $t$ and position $\boldsymbol{r}=(X, Y, Z)$ and frequency $v$, all as measured in the observer frame. The effects of synchrotron self-absorption at low frequencies are neglected. In the rest-frame of the plasma (where we denote observables with an apostrophe '), the synchrotron emissivity is given by:

$j_{v^{\prime}}^{\prime} \propto \mathcal{N}\left(B_{\perp}^{\prime}\right)^{\alpha+1}\left(v^{\prime}\right)^{-\alpha}$

(for instance: see Longair 2011). Here, $\mathcal{N}$ is proportional to the density of the relativistic particles (see appendix A, $B_{\perp}^{\prime}$ is the magnetic field strength perpendicular to the particle's velocity in the

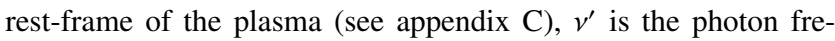
quency and $\alpha$, as before, the spectral index of the plasma.

In the observer frame, however, Doppler (de-)boosting needs to be taken into account. The synchrotron emissivity and the photon frequency are Doppler shifted according to (Begelman et al. 1984 and Rybicki \& Lightman 1986):

$j_{v}=\mathcal{D}^{2} j_{v^{\prime}}^{\prime}$

and

$v=\mathcal{D} v^{\prime}$

so that the emissivity observed at frequency $v$ is given by:

$j_{v} \propto \mathcal{D}^{2+\alpha} \mathcal{N}\left(B_{\perp}^{\prime}\right)^{\alpha+1}(v)^{-\alpha}$.

The Doppler factor $\mathcal{D}$ (for a smooth continuous jet) is given by:

$\mathcal{D}=\frac{1}{\gamma(1-\beta \cos (\psi))}=\frac{1}{\gamma(1-\boldsymbol{\beta} \cdot \boldsymbol{n})}$,

with $\gamma(\boldsymbol{r}, t)=1 / \sqrt{1-\boldsymbol{\beta} \cdot \boldsymbol{\beta}}$ the bulk Lorentz factor, $\beta(\boldsymbol{r}, t)=\boldsymbol{V}(\boldsymbol{r}, t) / c$ the bulk 3 -velocity of the plasma in units of $c$, and $\psi=\angle(\boldsymbol{n}, \boldsymbol{\beta})$ the angle between the line of sight and the bulk velocity of the plasma.

Based on a number of assumptions, we approximate various magnetic field configurations from the hydrodynamic quantities. Near the central engine of an AGN, magnetic fields are believed to have a helical structure (see for example Blandford \& Znajek 1977, Blandford \& Payne 1982, Keppens et al. 2008||McKinney \& Blandford 2009, Tchekhovskoy et al. 2011, Laing \& Bridle 2014, Zamaninasab et al. 2014, Gabuzda et al.2015: Prior \& Gourgouliatos 2019). At large distances from the central engine the large-scale magnetic field structures are much more difficult to determine: the way that the large-scale structures evolve is strongly dependent on jet dynamics (i.e. radial structure, velocity shear etc.); the conversion of magnetic energy into heat (i.e. magnetic reconnection); and interaction with the ambient medium, leading to back flow, vortices and jet pinches (see SW1).

The observed synchrotron emission from jets results from a line-of-sight integration of the synchrotron emissivity, which depends on the (probably complicated) field geometry, field strength and the distribution of the relativistic electrons. Therefore, based 
on the observations alone it is not possible to unravel the underlying field configuration. We make a sophisticated guess on how the magnetic pressure is distributed between the entangled field and the ordered (azimuthal, poloidal or helical) magnetic fields. Our heuristic method is explained in detail in Appendix $\mathrm{C}$

For the quantity $\mathcal{N}$, related to the density of relativistic particles, we use and compare two different models. The first model, which we shall refer to as the $\mathcal{N}$-Tracer model, makes extensive use of the tracers that are advected with each jet component. This model takes into account the energy losses due to adiabatic expansion, but neglects the effect of synchrotron cooling. The second model is referred to as the $\mathcal{N}$-Cooling model and is based on the work of Camus (2009), Section 4.3.4, and Del Zanna et al. (2006). It takes into account both the effects of adiabatic losses and synchrotron cooling. There, a power law of relativistic particles is injected along with the jet, characterised by a number density of relativistic particles, $n$, and a cut-off frequency $v_{\infty}$. The advantage of this model is that brightness variations are given more accurately at all observer frequencies, but this model is not able to separate the various contributions of different jet components. In the case where the observation frequency is much lower than the cut-off frequency $\left(v_{\mathrm{obs}}<<v_{\infty}\right)$, there is no significant synchrotron cooling. In this limit these two models should yield similar results, as we will show. The general expression for the emissivity 6 will be further specified in the next section for the $\mathcal{N}$-Tracer and the $\mathcal{N}$-Cooling models, of which a full derivation can be found in the appendices A B and C

\section{METHOD}

We have generated $2.5 \mathrm{D}$ simulations of cylindrically symmetric jets, based on similar simulations that we used in SW1 and SW2. For a complete explanation of the numerical method that was used for generating the simulations, we refer the reader to those papers.

We employ the code MPI-AMRVAC (see for example Keppens et al. 2012, Porth et al. 2014, Xia et al. 2018 or Teunissen \& Keppens 2019 and amrvac . org), and use the special relativistic hydrodynamical module (SRHDEOS) with the Mathews approximation for the Synge gas equation of state, suitable for a nonrelativistic gas, as well as a relativistically hot gas. Moreover, we use the Harten-Lax-van Leer-Contact solver (HLLC) (Toro, Spruce \& Speares 1994, Mignone \& Bodo 2005) combined with a Three step Runge-Kutta time-discretisation scheme and a Koren limiter (Koren 1993).

The base-level computational domain contains $(120 \times 480)$ grid cells, corresponding to the physical scale of $\left(120 \times 480 \mathrm{kpc}^{2}\right)$. We allow for three additional refinement levels, resulting in an effective resolution of $(960 \times 3840)$ grid cells. The jet is injected along the $Z$-axis at $Z=0$, between $R=0$ and $R=R_{\text {jet }}(Z=0)=$ $1 \mathrm{kpc}$. We study jets with three different radial profiles, namely a piecewise isochoric jet (model $A 2$ ); an isothermal jet (model $I 2$ ); and a homogeneous jet (model $H 2$ ). In this context, piecewise isochoric means that as the jet is injected into the system, the jet spine and the jet sheath are initiated with constant, but different mass densities. The jet spine is assumed to have a lower mass density than the jet sheath. We assume that at the jet inlet the jet spine and the jet sheath have already gone through some extent of turbulent mixing, compared to the conditions close to the AGN, so that their mass ratio is moderate. In correspondence with the previous papers SW1 and SW2, we have arbitrarily chosen the mass density ratio to be $\rho_{\mathrm{sh}} / \rho_{\mathrm{sp}}=5$.
The jets are switched on for 15.3 Myr; interrupted for 0.68 Myr; and restarted again for another 6.8 Myr, matching realistic time scales for DDGRs. For the jet in the initial phase (or simply "the first jet"), and the jet in the restarted phase (simply the "second jet") different tracers are advected. For the homogeneous jet $(H)$ this leads to two different tracers, but in the case of the radially structured jets (the $A$ or $I$ models) where we have a jet spine and a jet sheath, a total of four tracers is used in each simulation. In addition to the SRHDEOS module that was used for the simulations in SW1 and SW2, a new set of transport equations was added to the code in order to trace the number density and cut-off energy of a population of relativistic particles with a power law distribution of the form $\epsilon^{-s}$, see $\mathrm{A} 33$; $\mathrm{A} 34$ and $\mathrm{A} 35$.

The data files that are generated in the numerical simulations are used in the relativistic ray-tracer synchro.py (e.g. Porth, Komissarov \& Keppens 2014) in order to create the synthesized synchrotron maps. By reflecting the one-sided jet in the $Z=0$ plane (of the initial computational box), this jet is transformed into a twosided jet. The emissivity is then calculated in each grid cell of the new box. We choose the size of the new box to be $700 \times 700 \times 700$ $\mathrm{kpc}^{3}$ with a resolution of $300 \times 300 \times 300$ grid cells.

For $\vartheta=0^{\circ}$ the line of sight and the jet $Z$-axis are aligned (head-on), while for $\vartheta= \pm 90^{\circ}$ the line of sight is perpendicular to the jet axis (face-on). In our coordinate system positive viewing angles correspond to the upper jets (the NW jets in Fig 1 A) pointing towards the observer, while the lower jets (the SE jets in Fig 1 A) point away from the observer. For negative viewing angles this is the other way around, and corresponds to case of J1835+6204. We simulated the jet models with a large variety of viewing angles and find a best match with $\mathrm{J} 1835+6204$ for $\vartheta=-71^{\circ}$, which we will use in most cases, unless mentioned otherwise. The sky plane rotation angle is close to $\delta=30^{\circ}$, but we will use $\delta=90^{\circ}$ is most cases in order to favour the visibility of the synthesized synchrotron maps.

Here we compare the two synchrotron radiation models, the emissivity of the $N$-tracer model:

$j_{v} \propto \Sigma_{\mathrm{A}} \mathcal{D}^{2+\alpha} \mathcal{N}_{\mathrm{A} 0}\left(B_{\perp}^{\prime}\right)^{1+\alpha}(v)^{-\alpha}\left(\frac{\theta_{\mathrm{A}}^{\prime} \rho^{\prime}}{\rho_{\mathrm{A} 0}^{\prime}}\right)^{1+\frac{2}{3} \alpha}$,

where the sum runs over the various jet components (see appendix A1, and the emissivity of the $\mathcal{N}$-Cooling model:

$j_{v} \propto \mathcal{D}^{2+\alpha} \mathcal{N}_{0}\left(B_{\perp}^{\prime}\right)^{1+\alpha}(v)^{-\alpha}\left(\frac{n_{\mathrm{e}}^{\prime}}{n_{\mathrm{e} 0}^{\prime}}\right)^{1+\frac{2}{3} \alpha}\left(1-\sqrt{\frac{v^{\prime}}{v_{\infty}^{\prime}}}\right)^{2 \alpha-1}$,

(see appendix A3. Here $B_{\perp}^{\prime}$ is given defined through C28. After the intensity is calculated a Gaussian smoothening is applied with a FWHM of $8.0 \mathrm{kpc}$ to match a typical beam size of a radio telescope such as the VLA in B or D configuration at $4.8 \mathrm{GHz}$, with beam sizes of 1.14" -1.71 " as in the case of J1835+6204 (see Konar et al. 2012).

The brightness levels of the synthesized synchrotron maps are constructed as follows: After integrating the emissivity along the line of sight, the peak level radio flux $I_{v, \max }$ is determined per image. We let this flux level correspond to the peak flux level of the observed source J1835+6204. We choose a dynamic range of 256 from the peak intensity and show contours spaced by factors of two. This allows us to make a realistic comparison between the various synthesized synchrotron maps. 


\section{RESULTS AND DISCUSSION}

In this section we present the results of the two synchrotron radiation models that we have used for our synthesis maps. As a reference source, we will use the DDRG J1835+6204 observed at 4860.1 MHz (see Fig. 1 panel A). We will summarise the main features of $\mathrm{J} 1835+6204$ here.

J1835+6204 has two clearly distinct pairs of radio lobes: the outer North-Western and the South-Eastern lobes, referred to as NW1 and SE1, respectively; and the inner North-Western and the South-Eastern radio lobes (closest to the central engine situated at the plus-sign in the center of the image), referred to as NW2 and SE2, respectively. The viewing angle of the source is such that the SE lobes are pointing towards the observer and the NW lobes are pointing away from the observer. The outer radio lobes are thought to be caused by the first jet eruption; the space between the outer lobes and the inner lobes correspond to a quiescent phase in the activity of the central engine, where a jet was not injected; and the inner radio lobes are due to a second jet eruption. All four hotspots are visible and are well described by a power law, meaning that the hotspots are still being fed by the jets (or the time passed since the last material from the jet has reached the outer hotspot is relatively short). Based on the distance of the hotspots to the central engine and the jet-head propagation speed, it is possible to estimate the viewing angle of the radio jet (see for example Safouris et al. 2008 or Konar et al. 2013). We will briefly sketch this method here: When a radio galaxy ejects radio jets of which the jet axis is not exactly perpendicular to the line of sight, retardation effects come into play. The time it takes for light emitted by (for example the hotspot of the) approaching jet to reach the observer will be less than the time it takes for light emitted by the (hotspot of) receding jet, when we assume the jets to have equal physical size. This leads to a difference in the observed size of both jets. When the projected size of the approaching jet is $d_{\mathrm{a}}$, and the projected size of the receding jet is $d_{\mathrm{r}}$, the arm length ratio $D$ is then defined by:

$D=\frac{d_{\mathrm{a}}}{d_{\mathrm{r}}}=\frac{1+\beta_{\mathrm{hd}} \cos \vartheta}{1-\beta_{\mathrm{hd}} \cos \vartheta}$,

with $\beta_{\text {hd }}$ the jet-head advance speed, and $\vartheta$ the viewing angle, as before. This can be rewritten as:

$\cos \vartheta=\frac{1}{\beta_{\mathrm{hd}}} \frac{D-1}{D+1}$.

The projected linear sizes $d_{\mathrm{a}}$ and $d_{\mathrm{r}}$ for both the outer jets, as well as the inner jets for J1835-6204 are known (see Konar et al.|2012), and lead to an arm length ratio of $D \approx 1.02$. Moreover, the jet-head advance speed of the outer jets is typically of the order $\beta_{\text {hd }} \sim 0.03-$ 0.05 . The jet-head advance speed for the inner jets is usually much higher, and of the order $\beta_{\text {hd }} \sim 0.1-0.5$. Both cases agree very well with our simulations, as is discussed in SW2. Using these values, we find an estimate for the viewing angle $70^{\circ} \lesssim \vartheta \lesssim 89^{\circ}$. The inner hotspots are brighter by a factor of 2 compared to the outer ones for the NW jets and by a factor of 4 for the SE jets. The outer radio lobes are more elongated, while the inner radio lobes are more circular.

\subsection{Comparing J1835+6204 to models A2, I2 and H2}

In SW1 three radial jet profiles were explained in detail, namely:

- the homogeneous radial jet profile, denoted as ' $H$ '. This model describes a non-rotating structureless jet profile;

- the (piecewise) isothermal radial jet profile, denoted as ' $I$ '.
This model describes a jet that is initiated with an azimuthal velocity component, and constant temperature $T_{\mathrm{sp}}$ throughout the crosssection of the jet spine and constant (but possibly different) temperature $T_{\mathrm{sh}}$ throughout the jet sheath. We have chosen $T_{\mathrm{sp}}$ and $T_{\mathrm{sh}}$ to be equal in these simulations;

- and a (piecewise) isochoric jet profile denoted, as ' $A$ '. This model describes a jet that is initiated with an azimuthal velocity component, and constant (but different) mass densities throughout the cross-section of the jet spine and the jet sheath.

In SW2 the same radial profiles were used, but instead of the jets being continuously injected, there is a small period of intermittency where the central engine is not active. This interrupted jet flow leads to the behaviour as is observed in DDRGs. We refer to these jet models as $H 2, I 2$ and $A 2$, respectively.

\subsubsection{Similarities between the models A2, $\mathrm{I} 2$ and $\mathrm{H} 2$}

In Fig. 1]A, the radio image of J1835+6204 is compared to our best fit synthesized radio maps of jet models Fig. 11B: The (piecewise) isochoric jet model $A 2$; with sky plane rotation angle $\delta=30^{\circ}$; using the $\mathcal{N}$-Cooling synchrotron radiation model at an observation frequency $v_{\mathrm{obs}}=6 \cdot 10^{-4} v_{\infty}$; with 70 per cent of the magnetic pressure contributed by the entangled fields $(\Lambda=0.7)$ and 30 per cent contributed by ordered helical fields (pitch angle $\kappa=45^{\circ}$ ); and viewing angle $\vartheta=-71^{\circ}$.

In Fig. 2 A, B and $\mathrm{C}$, jet models $A 2 ; I 2$ and $H 2$ are shown respectively, with $\delta=90^{\circ}$ to improve visibility; a purely entangled magnetic field configuration $(\Lambda=1)$, and using the $\mathcal{N}$-Tracer synchrotron radiation model at $\vartheta=-71^{\circ}$. The interruption time for all three models is chosen to be 4.5 per cent of the injection time of the initial jet, in correspondence with $\mathrm{J} 1835+6204$ which is estimated to have an interruption of $\sim 1 \mathrm{Myr}$ and an active phase of the previous outburst of $\sim 22 \mathrm{Myr}$. The observations of J1835+6204 show that the ratio of the distance from the outer jet-heads to the AGN $\left(D_{\mathrm{jt}_{1}}\right)$ to the distance from the inner jet-heads to the AGN $\left(D_{\mathrm{jt}_{2}}\right)$ is approximately $D_{\mathrm{jt}_{1}} / D_{\mathrm{jt}_{2}}=7: 2$ (at $v_{\mathrm{obs}} \sim 4886 \mathrm{MHz}$ and a beam size of $\sim 1.4$ "). Our simulations match this ratio best at a time of 16.6 Myr, when the restarted jets have been injected for $\sim 0.6 \mathrm{Myr}$.

All three synthesized synchrotron maps globally show the same behaviour: the two pairs of hotspots are clearly visible at the termination shocks of the jets and counter jets, and a few additional radio blobs can be found along the jet axis. In all three models, the ratio between the distance from the central engine to the inner hotspots $\left(D_{\mathrm{hd} 2}\right)$ and to the outer hotspots $\left(D_{\mathrm{hd} 1}\right)$ is approximately the same: $D_{\mathrm{hd} 1} / D_{\mathrm{hd} 2} \approx 2-3$. The maximum intensity of the SE2 hotspots is clearly higher than that of the NW2 hotspots, whereas the NW1 hotspots in all three models are brighter than the SE1 hotspots. Finally: the bulk of the jet flow is not visible. Most of the emission comes from the hotspots, the cocoon surrounding the jet and some knots along the jet axis associated with instabilities in the jet/cocoon interface. At this dynamic range in intensity, the radio structure is broken up into distinct patches. Of the three jet models, the morphology of the isochoric jet model A2 compares best to the observed source $\mathrm{J} 1835+6204$.

\subsubsection{Differences between the models A2, I2 and H2}

The main differences between the three models are: [1] the shape of the (outer) hotspots; [2] the width of the surrounding cocoon (the radio lobes) and [3] the intensity contrast between the inner two 

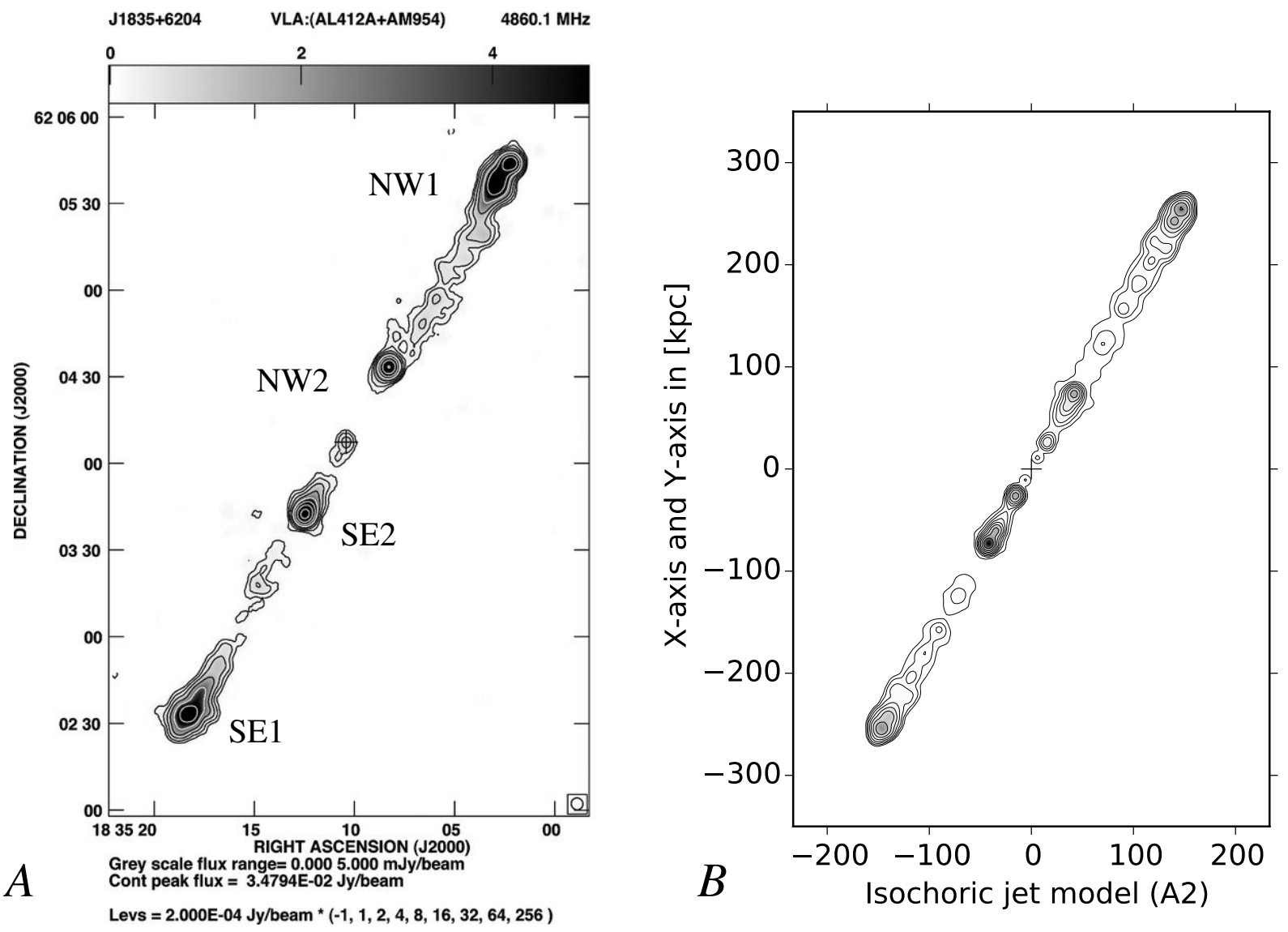

Figure 1. Comparison between the observed DDRG J1835+6204 and our best fit synthesized jet model A2. Panel A: The source J1835+6204 observed at 4860.1 MHz. The cross-hairs correspond to the AGN radio core at the center of the radio galaxy. (Credits: Konar et al. 2013 Fig. 2, p5). Panel B: The (piecewise) isochoric spine-sheath jet model (A2). The sky plane rotation angle $\delta=30^{\circ}$; the viewing angle $\vartheta=-71^{\circ}$; a faction of 70 per cent of the magnetic pressure contributed by entangled field $\Lambda=0.7$; using the $\mathcal{N}$-Cooling model at observation frequency $v_{\mathrm{obs}}=6 \cdot 10^{-4} v_{\infty}$. The contour levels for the synthesized synchrotron map have been chosen similar to the contours for the observed source J1835+6204. The flux levels are $I_{v} \in\{1,2,4,8,16,32,64,256\} \times I_{v, \text { min }}$, with $I_{v, \min }=I_{v, \max } / 256$ and $I_{v, \max }=1$. The cross hairs correspond to the jet-inlet of the simulation, located at a distance of $57 \mathrm{kpc}$ from the AGN for a jet with a $1^{\circ}$ opening angle, but at the inlet we choose the opening angle to be $0^{\circ}$.

hotspots and the outer two hot spots. The isochoric jet model $A 2$ shows almost round jet hotspots in both the inner and outer jets.

The outer hotspots of the isothermal jet model $I 2$ and of the homogenous jet model $\mathrm{H} 2$ appear to be more flattened in the direction perpendicular to the jet flow. The inner- and outer jets in the $I 2$ and $H 2$ models have a distinct morphology and are clearly separated in the synthesized synchrotron map. The inner jets of the $A 2$ jet model, on the other hand, lie partially within the radio structure of the outer jet radio structure, in good agreement with $\mathrm{J} 1835+6204$. In all cases we find this typical distinction between the $A 2$, the $I 2$ and the $H 2$ jet models.

\subsection{Influence of the radial profile of the jets}

Our previous studies (SW1 and SW2) focussed on the dynamical differences between the (piecewise) isochoric jet $(A)$ model, the isochoric jet $I$ model and the structureless homogeneous jet $H$ model. A strong structural integrity means that the jet is not easily affected by pressure fluctuations from the back-flowing jet material in the cocoon that surrounds the jet due to the formation of vortices, so that internal shocks along the jet axis are much less capable of disrupting the jet flow. Since the emissivity depends on the magnetic field strength, which in turn depends on the gas pressure for an entangled magnetic field configuration, radio features along the jet axis will become more apparent for jets with less structural integrity. This agrees well with the results in Fig. 11B and Fig. 2 A..

Our simulations show that the $H 2$ jet and the $I 2$ jet maintain their structural integrity well: interaction with the cocoon does not lead to a significant disruption of jet flow. In contrast: the $A 2$ jet suffers most disruption. This has a large influence on the radio morphology of these jets, as we discuss in the following subsections.

\subsubsection{The homogeneous jet model, the outer jets}

The homogeneous jet has the strongest structural integrity of the three models. Therefore, the homogeneous jet is less easily deformed in radial direction, so the jet flow will remain relatively close to the jet axis, in contrast to what happens in jets with a welldefined spine/sheath structure. This causes the Mach disc (termination shock at the jet-head) of the homogeneous jet to be less diffuse and more flat than the Mach discs of the structured jets, which have a larger surface area (the effective impact area) and have a bowl-shaped, elongated structure (see SW1 for more details on these jet-head structures). Therefore, the shock-heated gas for the homogeneous jet will be more concentrated at the jet-head and 
the synchrotron radiation generated in this part of the jet will outshine the other parts of the jet/cocoon structure.

As the jet material propagates from the jet inlet to the jet-head, it passed through $\sim 9$ internal shocks at this length scale for models $A 2, I 2$ and $H 2$. These internal shocks are caused by jet-cocoon interactions: pressure fluctuations in the cocoon for example by vortices that are created at the jet-head structure and flow away from the jet head, as was also discussed in SW1. From the simulations in SW1, a trending increase in temperature, pressure and relativistic heat (through the effective polytropic index) was found along the jet axis, clearly correlating to the passing of the jet material through internal shocks. From these simulations we found that the jet material was shocked to near-relativistic temperatures just ahead of the termination shock at the jet-head, and shocked to relativistic temperatures just after passing the termination shock. This explains the synchrotron intensity contours of the outer jets in Fig. 2 C: bright outer hotspots and no radio features from the outer cocoon, and small knots just ahead of the jet-heads.

\subsubsection{The spine-sheath jet models 12 and A2: the first outer jets}

The (piecewise) isothermal jet model $I$ assumes a constant temperature across a radial cut of the jet, but allows for the temperature of the jet spine and jet sheath to differ mutually. However, in order to minimise the number of free parameters, we've chosen the jet spine and sheath to have an equal temperature at the jet inlet, $T \sim 2 \times 10^{9} \mathrm{~K}$, which results from our choice of parameters (such as number density, kinetic luminosity of the jet, Lorentz factor of the bulk jet material), typical for FR-II jets and their ambient medium (see SW1 for a more detailed discussion). After passing the first internal shock, the temperature of the jet material is increased to $T \sim 10^{10}-10^{11} \mathrm{~K}$, in agreement with observations (e.g. Homan et al.2006 or Kellermann et al. 2007). This choice results in a continuous mass density profile at the jet spine/sheath interface. The isochoric jet model $A$, on the other hand, has a mass density contrast of a factor 5 at the spine-sheath interface at the jet inlet. Therefore, the isothermal jet is much more stable against the effect of pressure variations within the cocoon (from back flowing vortices) than the isochoric jet. As a result, the isothermal jet maintains more of its structural integrity than that of the (piecewise) isochoric jet as one moves further away from the central engine. The jet-head of the isochoric jet has a very elongated and bowl-shaped structure. Therefore, the region where the jet flow is shock-heated is larger and more diffuse, causing the radio structures of the jet-head (and surrounding back-flowing material) to be more extended than in the homogeneous and isothermal jet models. This explains the synchrotron intensity contours of the outer jets in panels B and C of Fig. 2. a relatively flat jet head from the isothermal jet, with radio features from the back-flowing material (and the Doppler boosting of back-flowing material is actually quite prominent); while the jet-head from the (piecewise) isochoric jet is more round and the surrounding back-flowing material is much better observed.

\subsubsection{The restarted, inner jets}

The inner (restarted, or second) jets of all three jet models $A 2, I 2$ and $H 2$ propagate through a much more dilute external medium: the disturbed intergalactic medium. It consists of a mixture of shocked jet material and shocked intergalactic material, with temperature, pressure and mass density comparable to that of the jet itself. The strength of the termination shock at the jet-head is de- termined by the (relativistic) Mach number: the ratio of the (relativistic) velocity of the jet-head as measured in the observer frame, and the (relativistic) sound velocity of the ambient medium (see SW2 for more details). This ratio will be significantly less for the restarted jets, because of the much higher temperature and low mass density of the material left behind by the first jet, so the Mach disc of the inner jets will not be as strong. The Mach number for the Mach disc of the outer jets for jet models $A 2, I 2$ and $H 2$ for Fig 2 are $\sim 13,22$ and 13 respectively, while the Mach numbers for the Mach disc of the corresponding inner jets are $\sim 8,16$ and 11, indeed a factor of $0.6-0.8$ as small for all three jet models. This is why the inner hotspots will be relatively less bright and do not outshine the surrounding jet material to the same extent as the hotspots in the first (older) jet that impact the intergalactic gas (this is why these inner jet-head radio features are also referred to as 'warm spots', see for example Konar et al. 2012). The general characteristics of these three jet models (as described in the previous section) will remain to be true, but more of the jet material along the jet axis will be visible, as compared to the first (older) jets. Finally, the jet-head advance speed strongly depends on the mass density ratio between jet material and ambient medium material, $\eta_{\mathrm{R}}=\rho_{\mathrm{jt}} / \rho_{\mathrm{am}}$. A small $\eta_{\mathrm{R}}$ (as is the case for jets that are under-dense compared to their ambient medium) leads to a smaller jet-head advance speed, while $\eta_{\mathrm{R}} \sim 1$ (for jets that have mass density comparable with that of their ambient medium, $\rho_{\mathrm{jt}} \approx \rho_{\mathrm{am}}$ ) leads to a higher jet-head advance speed (see SW2). The inner jets in the models $A 2, I 2$ and $H 2$ have mass density ratio very close to $\eta_{\mathrm{R}}=1$, so they propagate much faster through the cocoon that was left behind by the first jets, than the propagation speed of the first jets themselves, which have $\eta_{\mathrm{R}} \approx 0.01$, despite their lower Mach numbers. Therefore, the discharge of jet material through the Mach disc of the inner jets will be much smaller, leading to a back-flow that is also less strong. As a result, the inner jets encounter less pressure variations, and maintain their integrity better than the outer jets. The structured spine-sheath jets have a higher Lorentz factor jet spine. In case of the inner jets, this high Lorentz factor jet material will be able to propagate all the way up to the jet-head. This is why the effect of Doppler (de-)boosting is so clearly seen for the inner jets in all three models. In this work we are trying to explain the radio morphology of a DDRG such as J1835+6204. We find that the only possible jet model that reproduces its resolution-frequency specific view from Fig. 1, is the (piecewise) isochoric jet model $A 2$. Therefore, we continue with this model for further examination of the various parameters.

\subsection{Comparing various magnetic field configurations}

In this section we focus on the main differences between the different magnetic field configurations and which magnetic field configuration(s) we expect to be most realistic. In the simulations at this dynamical range, the purely structured magnetic fields appear as knots along the jet axis. Here, we have assumed the structured magnetic fields outside the jets (within the cocoon) to be so small (in fact, absent) compared to the fields inside the jet flow that they can be ignored.

The general behaviour of the radio features caused by structured magnetic fields within the jets can be explained as follows: For the sake of simplicity of the argument we assume the Doppler factor to be small. Then the line of sight vector measured in the observer frame, $\boldsymbol{n}$, and as measured in the plasma rest-frame, $\boldsymbol{n}^{\prime}$, are equal. In that case, the magnetic field component perpendicular to the line of sight, $B_{\perp}$, is equal in both frames. Then, when a 

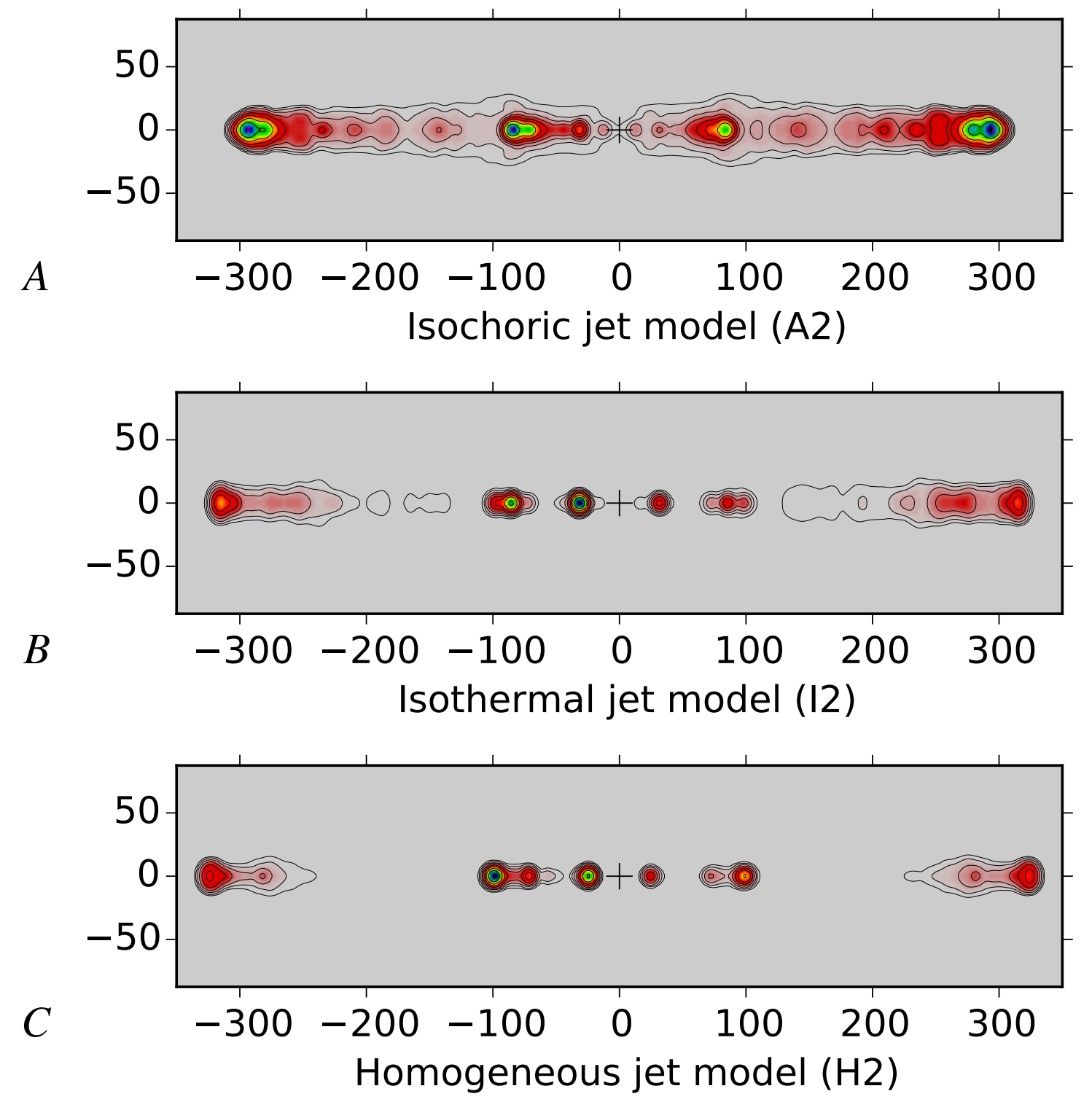

Figure 2. Panels A, B and C show the jet models $A 2, I 2$ and $H 2$ with the sky plane rotation angle $\delta=90^{\circ}$ and a continuous color palet for better visibility compared to Fig. 1 B. For these images, the $\mathcal{N}$-Tracer model was used, with viewing angle $\vartheta=-71^{\circ}$ and a purely entangled field configuration, corresponding to $\Lambda=1$. Panel A: The synthesized synchrotron map of the isothermal spine-sheath jet model $(A 2)$. Panel B: The synthesized synchrotron map of the isothermal spine-sheath jet model (I2). Panel C: The synthesized synchrotron map of the homogeneous structureless jet model $(H 2)$. The flux levels are $I_{v} \in\{1,2,4,8,16,32,64,256\} \times I_{v, \min }$, with $I_{v, \min }=I_{v, \max } / 256$ and $I_{v, \max }=1$.

radio jet is viewed face-on (so the line of sight is perpendicular to the jet axis), the magnitude of the perpendicular field component of the poloidal field is at its maximum, while the magnitude of the azimuthal field is at its minimum. Therefore, one can expect a larger contribution to the perpendicular field component coming from the poloidal field than from the azimuthal field. The opposite is true when the jet is viewed head-on (so when the line of sight vector coincides with the direction of the jet-axis). In most of the synthesized synchrotron images in this paper, the jets have a viewing angle of $\vartheta=-71^{\circ}$, tending toward a face-on situation. This might explain why the synthesized synchrotron images from the poloidal field configuration are more prominent than those of the azimuthal field configuration (as discussed in the next alinea). In Fig. 3 the structured jet model A2 is shown in the case where the magnetic fields are purely ordered $(\Lambda=0$, see appendix $\mathrm{C} 4$. Since we assume these ordered fields are confined to the jets, the synchrotron emission has a very thin, elongated morphology. Panel A assumes a purely azimuthal magnetic field (where the pitch angle $\kappa=0^{\circ}$ ), whereas panel B assumes a purely poloidal magnetic field (where the pitch angle $\kappa=90^{\circ}$ ). We note that for these images, the position of the outermost knots in the synchrotron radiation is just ahead of the termination shock of the jets (relative to the central engine). This means that in case of the purely ordered fields, the termination shock itself does not contribute the most to the radio emission. An explanation for this effect is that the ordered fields are strongest where the jet radius is pinched the most. This occurs just ahead of the termination shock due to high-pressure shock-heated backflowing jet material in the surrounding cocoon. 

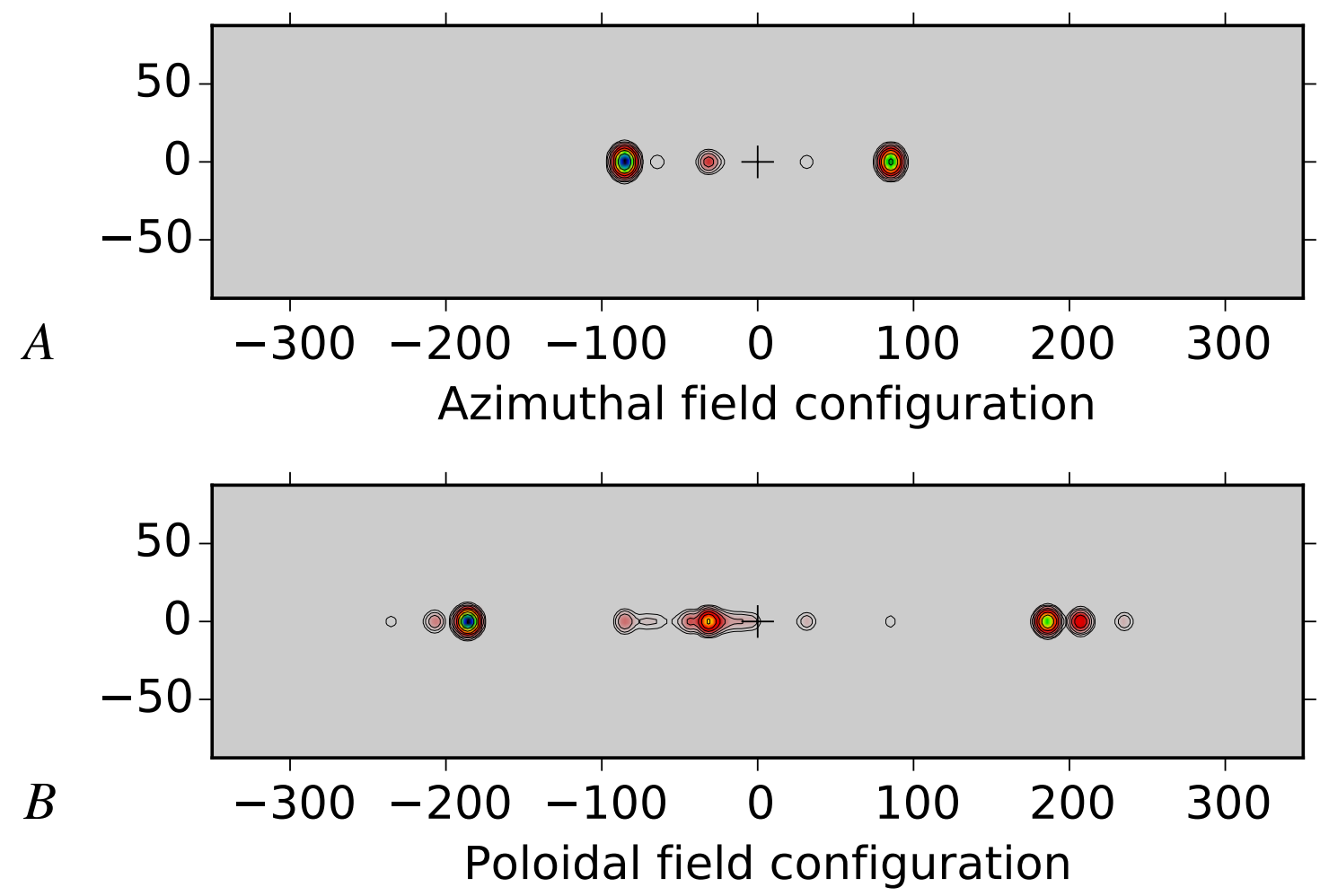

Figure 3. Purely ordered magnetic field configurations for the structured isochoric jet model $A 2$. The entangled magnetic fields are absent and the ordered magnetic field are assumed to exist only within the jets. Panel A assumes a pure azimuthal field configuration (so $\Lambda=0$ and $\kappa=0^{\circ}$ ); Panel B assumes a pure poloidal field configuration (so $\Lambda=0$ and $\kappa=90^{\circ}$ ); The flux levels are $I_{v} \in\{1,2,4,8,16,32,64,256\} \times I_{v, \min }$, with $I_{v, \min }=I_{v, \max } / 256$ and $I_{v, \text { max }}=1$.

\subsubsection{Structured magnetic fields for model A2}

Fig. 3 A shows the A2 jet for a purely azimuthal magnetic field configuration $\left(\boldsymbol{B}=\boldsymbol{B}_{\phi}\right)$. Only the emission from the restarted jets can be seen in this image, meaning that the contribution from the initial jets is at least a factor of 256 smaller than the peak brightness level in the image. The radio structure is not continuous along the jet axes, but just shows a number of knots (three on each side). These knots are a result of the jet being locally compressed by external pressure fluctuations. Such compressions cause the jet radius $R_{\mathrm{jt}}$ to reduce and since $B_{\phi} \propto R / R_{\mathrm{jt}}^{2}$, a local compression of jet radius causes a local increase in magnetic field strength. As a result, the emissivity is also enhanced. These local maxima in synchrotron emissivity appear as knots (for the dynamical range in radio emission chosen in this paper). Each knot from the SE2 jet is brighter than the corresponding knot on the NW2 jet. The Lorentz factor of these inner jets is relatively high. Therefore, the brighting of the (approaching) SE2 radio features compared to the corresponding (receding) NW2 jets is due to Doppler boosting/dimming.

Fig. 3B shows the A2 jet for a purely poloidal magnetic field configuration $\left(\boldsymbol{B}=\boldsymbol{B}_{Z}\right)$. In contrast to the azimuthal case, here the contribution from the initial jets is most clearly visible. The contribution from the NW2 jet is virtually absent. The same argument used to explain the appearance of knots in the azimuthal case holds for the poloidal case, with the only difference that the magnitude of $B_{Z}$ scales as $B_{Z} \propto 1 / R_{\mathrm{jt}}^{2}$. The two knots that are the brightest in the SE2 jet in the azimuthal case are also seen in the poloidal case, but they are significantly weaker than outermost knots. The outer knots from jet NW1 are slightly brighter than the corresponding SE1 jets.

\subsubsection{Mixed magnetic fields for the model A2 with $\Lambda=0.5$}

Fig. 4 shows the images resulting from mixed turbulent (entangled) and regular (ordered) magnetic field configurations (see C4 for more information). Panels A and B show the images where the (maximum) energy of the entangled field equals the (maximum) energy of the ordered magnetic fields, corresponding to $\Lambda=0.5$.

Panel A shows the case where the ordered fields are azimuthal, and panel B shows the case where the ordered fields are poloidal. The effect of adding the azimuthal field to the entangled field is that the inner radio structure (from the restarted jets) and the outer radio structures (from the initial jets) break up. The overall characteristics remain, however, the relative brightness of the outer radio features is significantly less than in the case with the purely entangled fields. Adding a poloidal field to the entangled field leads to less obvious changes in the synthesized map. The inner and outer radio structures do not break up in this case and the brightness contrasts between inner and outer jets (NW2 v.s. NW1 and SE2 v.s. SE1) do not change significantly. Close examination shows that knots between the hotspots become slightly brighter.

\subsubsection{Mixed magnetic fields for the model A2 with helical fields}

Panels C and D of Fig. 4 show the case of a mixed field configuration, where the ordered magnetic fields are helical with a fixed pitch angle $\kappa=45^{\circ}$. In panel $\mathrm{C}$, the (maximum) magnetic energy from the entangled fields is one third of the (maximum) magnetic energy from the ordered magnetic field, so $\Lambda=0.25$. In this case, the contribution from the initial jets NW1 and SE1 to the total brightness becomes less significant and their radio features become less 

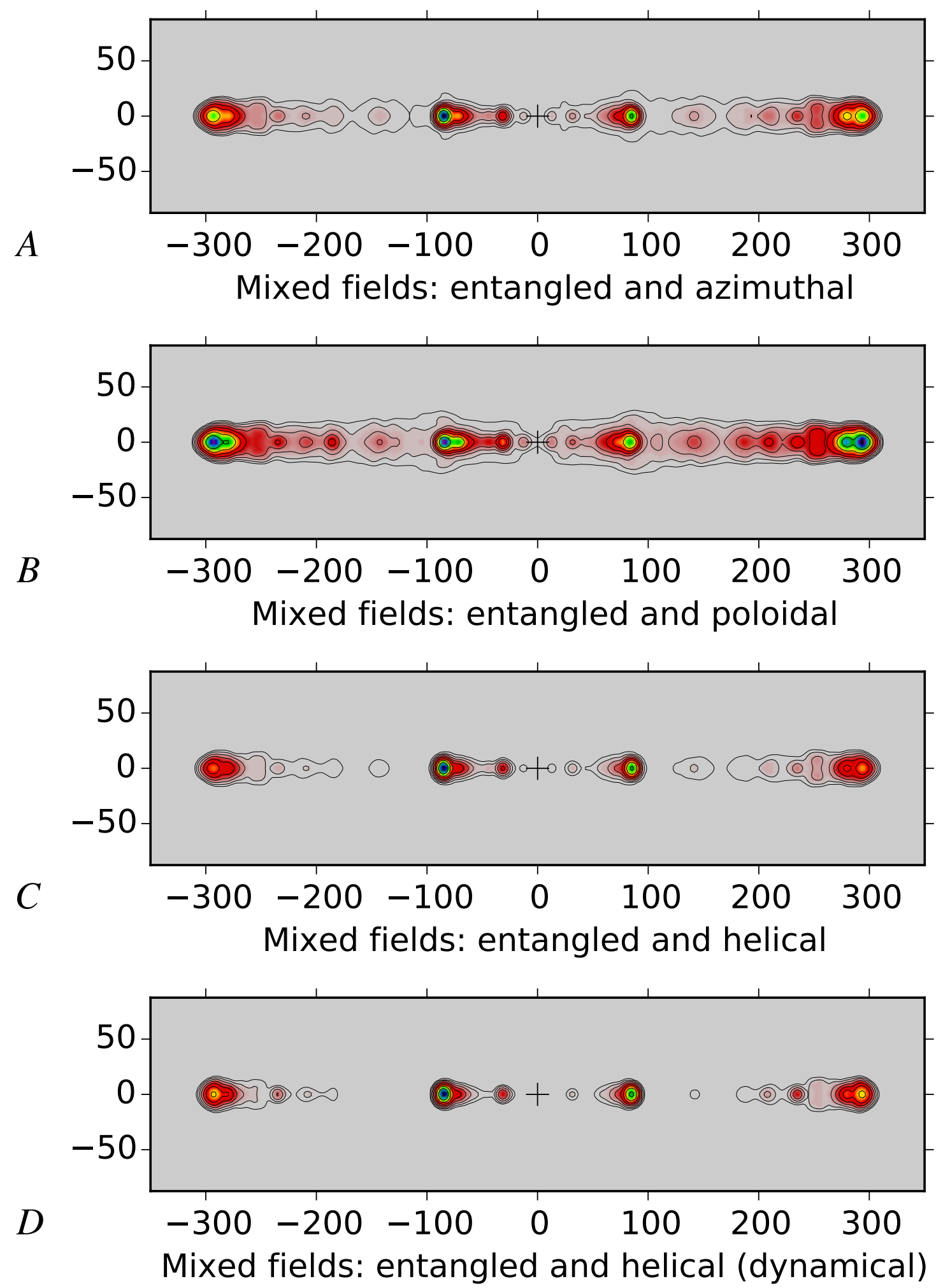

Figure 4. Various mixed magnetic field configurations for the isochoric jet model (A2): In panels A end B the magnetic pressure of the entangled fields in the jets is half of the total magnetic pressure (so $\Lambda=0.5$ ). Panel A: within the jets, an azimuthal magnetic field configuration is assumed (so $k=0^{\circ}$ ). Panel B: within the jets, a poloidal magnetic field configuration is assumed. (so $K=90^{\circ}$ ). Panel C: within the jets, a helical magnetic field configuration is assumed with $\kappa=45^{\circ}$. The magnetic pressure of the entangled fields is now chosen to be one quarter of the total magnetic pressure (so $\Lambda=0.25$ ). Panel D: also here a helical magnetic field configuration is assumed with $K=45^{\circ}$, however, in this case we use the absolute mixing factor $\Lambda_{\mathrm{AB}}$ for the fraction of the magnetic pressure. The flux levels are $I_{v} \in\{1,2,4,8,16,32,64,256\} \times I_{v, \min }$, with $I_{v, \min }=I_{v, \max } / 256$ and $I_{v, \max }=1$. 
elongated. Radio features between the inner hot spots NW2 and SE2, and the outer hotspots NW1 and SE1 have almost vanished. In panel $\mathrm{D}$, a model is shown where the magnetic energy fraction $\Lambda$ is not a fixed number between 0 and 1 , but is actually equal to the mass-weighted mixing factor $\Lambda_{\mathrm{AB}}$ (see SW1 and SW2). This quantity calculates to what extent the spine and sheath of a structured jet have mixed and therefore could be a measure for the amount of entanglement of the magnetic fields. Comparing this image to panel $\mathrm{C}$, we notice quite a lot of similarities with respect to brightness contrasts, shape of the radio features, the number of knots, etc. Therefore, if the mass-weighted mixing factor $\Lambda_{\mathrm{AB}}$ were to be used as a measure of the entanglement, it would roughly correspond to $\Lambda \sim 0.25$. If these simulations represent a realistic scenario, then the overall radio morphology of a typical DDRG is clearly dominated by an entangled field configuration. At the physical length scales that we are simulating, it is reasonable to assume that the structured magnetic fields have been entangled significantly due to turbulence and passing multiple internal shocks. The knots as a result of a pinched jet radius for the structured magnetic fields appear just before (so upstream of) the actual internal shocks along the jet axis, since the jet radius has a local minimum there. The knots as a result of reduced jet radius for the entangled magnetic fields on the other hand appear just after (so downstream) of the internal shocks, since the gas pressure (which is assumed to be close to equipartition with the magnetic pressure) has a local maximum. Through the observation of such sources at radio frequencies, information about the local field configuration can be gained, and can be checked with polarization measurements.

\subsection{Effects of spectral ageing}

Spectral ageing is a crucial aspect to consider, since radiative losses determine whether or not one can see the outer lobes in radio maps, vital to know in the area of wide area radio surveys (like LoTSS, Shimwell et al. 2019). Generally speaking, spectral ageing of the relativistic electrons allows one to estimate the age of the source, or - if no ageing is seen - to infer the need for re-acceleration inside the jets or radio lobes.

In Fig. 5 we show the effect on the radio maps of synchrotron losses of the relativistic electrons responsible for the emission (spectral ageing, see A3 for more details). The value of the cutoff frequency is evaluated at each grid cell and has arbitrary units, since in our model we just study the brightness contrasts in the synthesized images. Therefore, we can choose $v_{\infty}=1$ at the jetinlet. The actual break frequency for $\mathrm{J} 1835+6204$ is approximately $2.3 \cdot 10^{4}<v_{\infty}<5.9 \cdot 10^{5} \mathrm{MHz}$, with an average of $v_{\infty}=3.1 \cdot 10^{5}$ $\mathrm{MHz}$ (Konar et al. 2012). As the material in the jets propagates towards the jet-heads, the jets expand sideways. As a result the cutoff frequency decreases as one moves outwards from the central engine. At the jet-head, kinetic energy from the jet material is converted to gas pressure, resulting in a high-temperature gas that flows back in the direction of the central engine, forming the cocoon that surrounds the jet. The pressure gradients between the cocoon material and the undisturbed intergalactic medium in turn also causes the cocoon to expand sideways, away from the jet-head. As a result, the brightness of the DDRG should also decrease for observation frequencies $v_{\mathrm{obs}}$ close to the cut-off frequency. This effect is clearly visible in Fig. 5 Panel A shows the DDRG for an observation frequency $v_{\mathrm{obs}}=1 \times 10^{-1} v_{\infty}$ (corresponding to $3.1 \cdot 10^{1}$ $\mathrm{GHz}$ ). In this case, the outer hotspots are just visible (since the outer jets and the surrounding cocoon have expanded quite significantly), whereas the inner jets are relatively bright (since the inner jets, em- bedded in the high-pressure gas of the first jet, have not expanded that much laterally). Panel B shows the DDRG at an observation frequency $v_{\mathrm{obs}}=1 \times 10^{-2} v_{\infty}$. In this case, the outer hotspots are much more prominent again, but the contours of the surrounding cocoon are still largely absent. As the observation frequency is decreased, more and more of the surrounding cocoon will become visible. In our study we find that the radio structures virtually do not show any effects of synchrotron cooling for observation frequencies $v_{\text {obs }} \lesssim 1 \times 10^{-6} v_{\infty}$ (corresponding to $\sim 0.3 \mathrm{MHz}$ ). To make a fair comparison between the $\mathcal{N}$-Cooling model (which includes the effects of spectral ageing) and the $\mathcal{N}$-Tracer model (which does not include spectral ageing) we have to consider the DDRG with the $\mathcal{N}$-Cooling model at sufficiently low observation frequency. These numbers imply that for our reference radio image of J1835+6204 at $4.8 \mathrm{GHz}$, corresponding to $v_{\mathrm{obs}} \sim 1.5 \times 10^{-3} v_{\infty}$ spectral ageing effect would be notable, but would not yet drastically change the morphology of the DDRG. The image at panel $\mathrm{C}$ shows the DDGR for an observation frequency of $v_{\mathrm{obs}}=1 \times 10^{-18} v_{\infty}$, where the complete radio structure is visible. Panel D shows the DDRG in case of the $\mathcal{N}$-Tracer model (arbitrary observation frequency). Close examination of the two bottom figures shows virtually no difference in any of the radio structures. This strongly indicates that the $\mathcal{N}$-Cooling and $\mathcal{N}$-Tracer synchrotron models converge at sufficiently low observation frequencies.

\subsection{Contributions from various jet components}

There are strong indicators that astrophysical jets emerging from an AGN of a radio galaxy have a transverse radial structure (see for instance Sol, Pelletier \& Asseo 1989, Aloy et al. 2000, Giroletti et al. 2004, Ghisellini, Tavecchio \& Chiaberge 2005, Gómez et al. 2008, Fuentes et al. 2018|Martí|2019, Park et al.|2019). These jets are believed to consist of a low-density, high-Lorentz factor jet spine, surrounded by a denser, slower moving jet sheath. This leads to two distinct jet components, each of which is given a separate tracer in the $\mathcal{N}$-Tracer model. Since we would like to keep track of each of the various jet components, we assign a total of four tracers to the $\mathcal{N}$-Tracer jet model: $\theta_{\mathrm{sp}_{1}}, \theta_{\mathrm{sh}_{1}}, \theta_{\mathrm{sp}_{2}}$ and $\theta_{\mathrm{sh}_{2}}$. The $\mathcal{N}$-Tracer model allows one to include the contribution of any separate jet component to the total emissivity, in any desired ratio. Therefore, it's possible to isolate the contribution of single jet components, or the combination of multiple jet components to the total emissivity.

Fig. 6 shows the jets at an age of 16.6 Myr (as before), in case of the $\mathcal{N}$-Tracer model. We've assumed a purely entangled magnetic field configuration (so $\Lambda=1$ ). We show the contribution of the initial jets to the total emission $\left(\theta_{\mathrm{sp}_{1}}\right.$ and $\left.\theta_{\mathrm{sh}_{1}}\right)$ in panel A; the contribution of the restarted jets to the total emission $\left(\theta_{\mathrm{sp}_{2}}\right.$ and $\left.\theta_{\mathrm{sh}_{2}}\right)$ in panel B; the contribution of the jet spine material to the total emission $\left(\theta_{\mathrm{sp}_{1}}\right.$ and $\left.\theta_{\mathrm{sp}_{2}}\right)$ in panel $\mathrm{C}$; and the contribution of the jet sheath material to the total emission $\left(\theta_{\mathrm{sh}_{1}}\right.$ and $\left.\theta_{\mathrm{sh}_{2}}\right)$ in panel $\mathrm{D}$.

In Fig. 6A, the contribution of the initial jets is isolated. The two outer hotspots are clearly visible, and the inner jets are absent. Moreover, the structure of the cocoon and radio lobes shows little differences with that of the complete image that includes the contribution of both the initial jets and the restarted jets. Surprisingly, we see that there are two very clear 'warm spots' just ahead of where the termination shock of the second jets should be. Apparently, leftover jet material from the first jet eruption is locally compressed so much by the head of the restarted jet that it significantly contributes to the total emissivity in the synthesized map.

In Fig. 6 B, the contribution of the restarted jets is isolated. Since these restarted jets have not propagated that far along the axis 

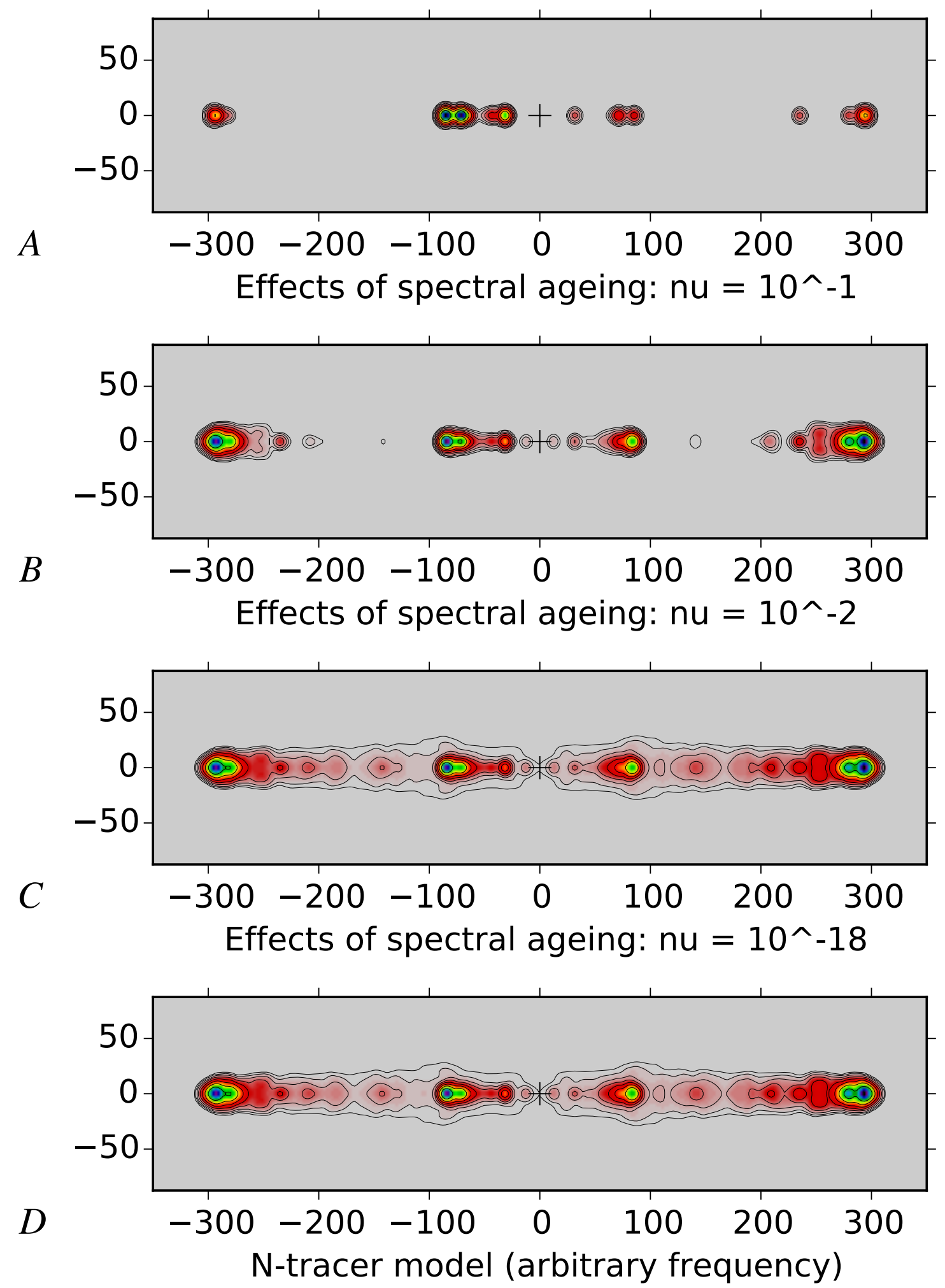

Figure 5. Maps showing the influence of spectral ageing. The cut-off frequency at the inlet of the jet is chosen to be $v_{\infty}=1$ (in arbitrary units), whereas the floor value of the cut-off frequency is chosen to be $v_{\infty}=1 \times 10^{-18}$. Panel A: synchrotron map for $v_{\mathrm{obs}}=1 \times 10^{-1} v_{\infty}$. Panel B: synchrotron map for $v_{\mathrm{obs}}=1 \times 10^{-2} v_{\infty}$. Panel C: synchrotron map for $v_{\mathrm{obs}}=1 \times 10^{-18} v_{\infty}$. These three images created by using the $\mathcal{N}$-Cooling model. Panel D: synchrotron map for the $N$-tracer synchrotron model, with arbitrary frequency (no spectral ageing applies). The flux levels are $I_{v} \in\{1,2,4,8,16,32,64,256\} \times I_{v, \text { min }}$, with $I_{v, \min }=I_{v, \max } / 256$ and $I_{v, \text { max }}=1$. 

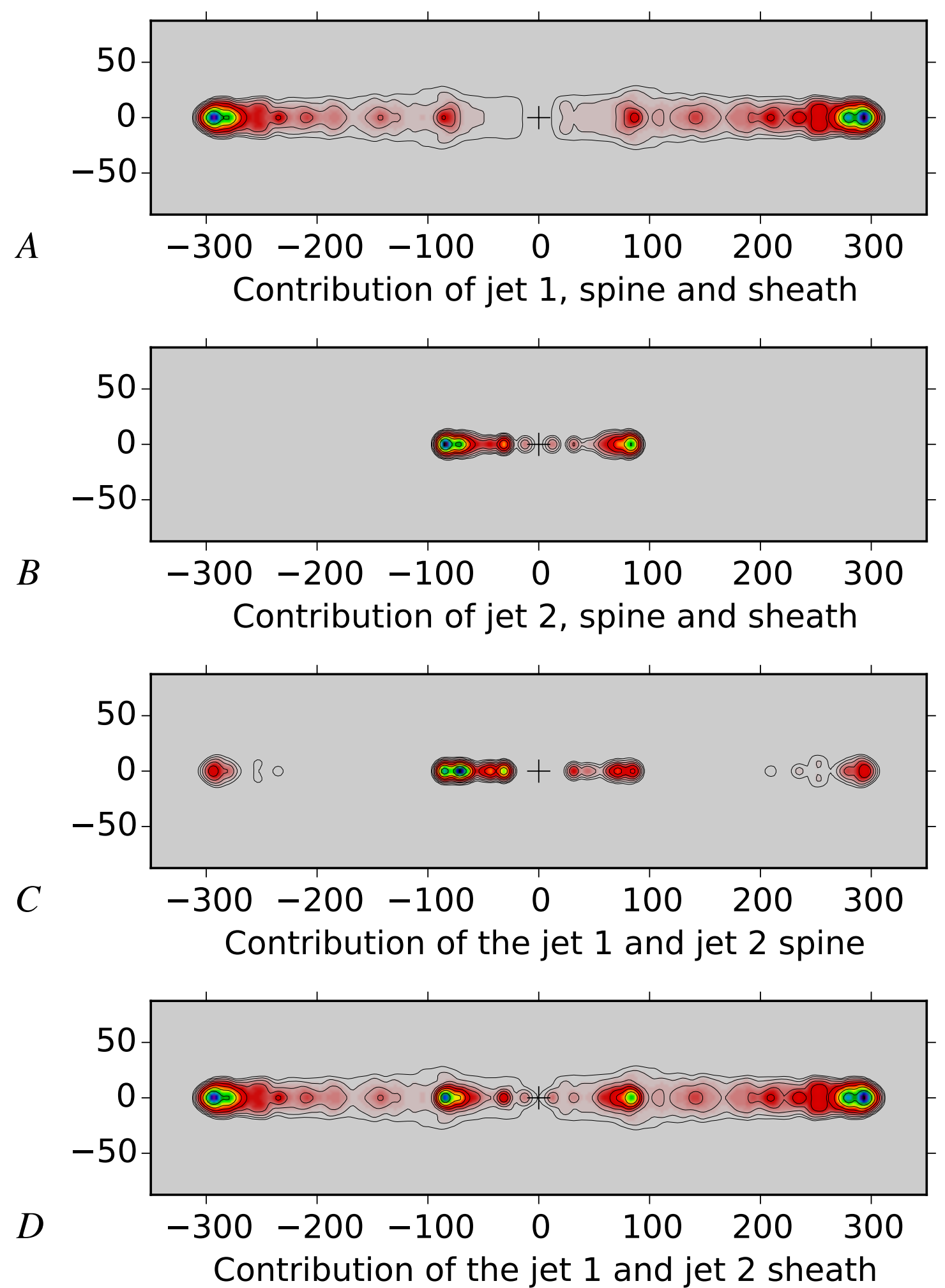

Figure 6. Contributions coming from the various jet components in the case of purely entangled magnetic fields (so $\Lambda=1.0$ ). Panel A: contribution to the total synchrotron emission coming from the initial jet (jet 1 spine and sheath). Panel B: contribution to the total synchrotron emission coming from the restarted jet (jet 2 spine and sheath). Panel C: contribution to the total synchrotron emission coming from the jet spine material (from jet 1 and 2). Panel D: contribution coming from the jet sheath material (from jet 1 and 2). The flux levels are $I_{v} \in\{1,2,4,8,16,32,64,256\} \times I_{v, \min }$, with $I_{v, \min }=I_{v, \max } / 256$ and $I_{v, \max }=1$. 
of the system, the associated radio features are relatively compact, much thinner, and the restarted jets are much more stable. This is reflected in the image: the cocoon is localised near the jet axis and more of the jet itself is visible.

Fig. 6 $\mathrm{C}$ shows the contribution of the jet spine material of both the initial jets and the restarted jets to the total emission of the synthesized maps. First of all, the radio features of the spine of the restarted jet (shown here together with the spine of the initial jet) seem somewhat thinner than panel B, where the full (spine+sheath) restarted jet is shown. This indicates that the restarted jet maintains its radial structure. For the initial jet, most of the lobe material is not seen in this map, except for a small contribution to the total emission in the outermost hotspots of the initial jets. This implies that almost all of the radio structures of the initial jets is due to the jet sheath material.

Looking at Fig. 6D, this suspicion is confirmed. There, the image shows the contribution of the jet sheath material of the initial jets and of the restarted jets. This image shows great similarities with the image of the total emissivity, in fact, the radio structures of the outer radio lobes is almost identical. It is clear that the brightness contrasts of the inner jets differ from the brightness contrasts in the map of the total emissivity: the inner jets seem to be less continuous and the inner structures are less bright.

\subsection{Effects of viewing angle}

In Fig. 7 the effect of a changing viewing angle is shown. Panel A shows a viewing angle of $\vartheta=-30^{\circ}$; Panel B shows a viewing angle of $\vartheta=-45^{\circ}$; panel $\mathrm{C}$ shows a viewing angle of $\vartheta=-60^{\circ}$; and panel D shows a viewing angle of $\vartheta=-89^{\circ}$ (so almost face on, but still slightly tilted to break the symmetry between the NW and SE jets).

The observed size of the DDRG $D_{\text {obs }}$ is determined by the actual size of the source, $D_{\text {DDRG }}$, multiplied by $\sin \vartheta$, so the smaller the viewing angle, the smaller the observed size of the source. The viewing angle has an effect on two quantities, namely [1] the Doppler factor and [2] the perpendicular magnetic field component of an ordered magnetic field configuration. Below we only discuss the effects of the viewing angle for jets with an entangled field configuration. This means that a change in the features is completely attributed to a change in the Doppler factor, assuming that the coherence length of the turbulent magnetic field is much smaller than the scale corresponding to the resolution adopted in these images.

The observed synchrotron emissivity at a certain frequency $v$ is very sensitive to variations in the velocity and viewing angle through the Doppler factor, as given by 6 . Since the inner jets propagate through a much more dilute medium than the outer jets, their jet-head advance speed (and therefore also advance speed of the hotspots) is much larger. In our models we find for the outer jet-head $\beta_{\mathrm{hd}_{1}} \approx 0.045$, while for the inner jets we find $\beta_{\mathrm{hd}_{2}} \approx 0.7$, roughly a factor of 16 times larger (see SW2). These values compare quite well to those inferred from observations, such as J1835+6204 (see for example Konar et al.2012). Based on these values, we find that the Doppler factor of the approaching inner jet is larger than that of the approaching outer jet for viewing angles $-65^{\circ}<\vartheta<0^{\circ}$. For $-20^{\circ}<\vartheta<0^{\circ}$, the ratio of the Doppler factor of the inner jet to that of the outer jet is even more than a factor of $\mathcal{D}_{\text {in }} / \mathcal{D}_{\text {out }}>2$. As a consequence the observed synchrotron emission, which scales as $D^{2+\alpha}$, can be a factor of 5-10 times as high as for the outer jets. As a result, when the source is tilted toward the observer so that the viewing angle becomes smaller, the synchrotron emission of the approaching inner jet is able to out- shine its corresponding outer jet. This can best be seen in panel A where $\vartheta=-30^{\circ}$. There the SE2 (inner) jet is significantly Doppler boosted, while the NW2 (inner) jet is so Doppler dimmed that it is almost no longer visible. Moreover, both the (outer) NW1 and SE1 jets are very weak compared to, and are outshined by, the SE2 jet.

The brightness contrast between the outer NW1 and SE1 jets is not that big, which implies that the Doppler (de-)boosting is not strong for these jets. In fact, close examination shows that the NW1 hotspot is actually brighter than the SE1 jet. This can only be explained by the effect of the Doppler factor associated with the backflowing material in the cocoon of the receding jet. This opens the possibility that a telescope with a limited dynamic range does not observe the outer hotspots or radio features of a DDRG at all if the viewing angle of that source is too small.

The general effects of the Doppler (de-)boosting on the morphology of the DDRG is observed at a wide range of viewing angles. Its effect becomes less strong for viewing angles near $\pm 90^{\circ}$. At $\vartheta=-45^{\circ}$ the outer and inner jets are still completely disconnected, but at $\vartheta=-60^{\circ}$ the outer NW1 contour encloses the NW2 jet. With large viewing angles both inner jets are enclosed by their outer counterparts, as can be seen in panel D.

\subsection{Comparing different stages of evolution}

The jet-head advance speed of the restarted jets is much higher than that of the initial jets. In fact, the restarted jets only reside within the outer cocoon for a small fraction of time, compared to the age of the source. In Fig. 8 we show a few important phases of the evolution of a DDRG. Panel A shows the DDRG at a time of $16.0 \mathrm{Myr}$, where the initial jets have been switched off for approximately $7 \times 10^{5}$ years. The hotspots are still being fed by the initial jets that have not yet dissipated. This observation was also made in the previous paper SW2, where a clear tail of jet material with Lorentz factor well above 1 could be seen. Even though the tails of the jets have moved outwards compared to the central engine, radio features are still visible close to the central engine. At $16.0 \mathrm{Myr}$, the restarted jets are injected and the inner jets propagate through the cocoon of the initial jets, while the leftover initial jets still feed the outer hotspots. This phase continues until the tails of the initial jets reach the outer hotspots, when a new phase starts: In panel B, the DDRG is shown at a time of 16.65 Myr, just after the initial jets have dissipated and the outer hotspots are no longer fed by jet material. The outer cocoon is still clearly visible, but its brightness weakens as the system expands adiabatically. At a certain point the restarted jets will reach the edge of the outer cocoon. This boundary of the outer cocoon is a relatively thick shell (whose density can be a factor $>4$ more than that of the undisturbed intergalactic medium). This means that as soon as the restarted jets start to interact with the outer cocoon a very strong termination shock forms. At this point, all other radio features that are contained within the cocoon are outshined by the newly formed termination shocks and only two very bright hotspots will be visible. This situation is shown in panel C of Fig. 8 at a time of 18.0 Myr. Because the shell of the (outer) cocoon is so dense, it takes the inner jets quite a long time to completely penetrate them, in our simulations approximately $7 \times 10^{5}$ years. The back-flow of jet material in this phase is also relatively strong. Panel D shows the system at $18.7 \mathrm{Myr}$, just after the restarted jets have just penetrated the outer cocoon. 

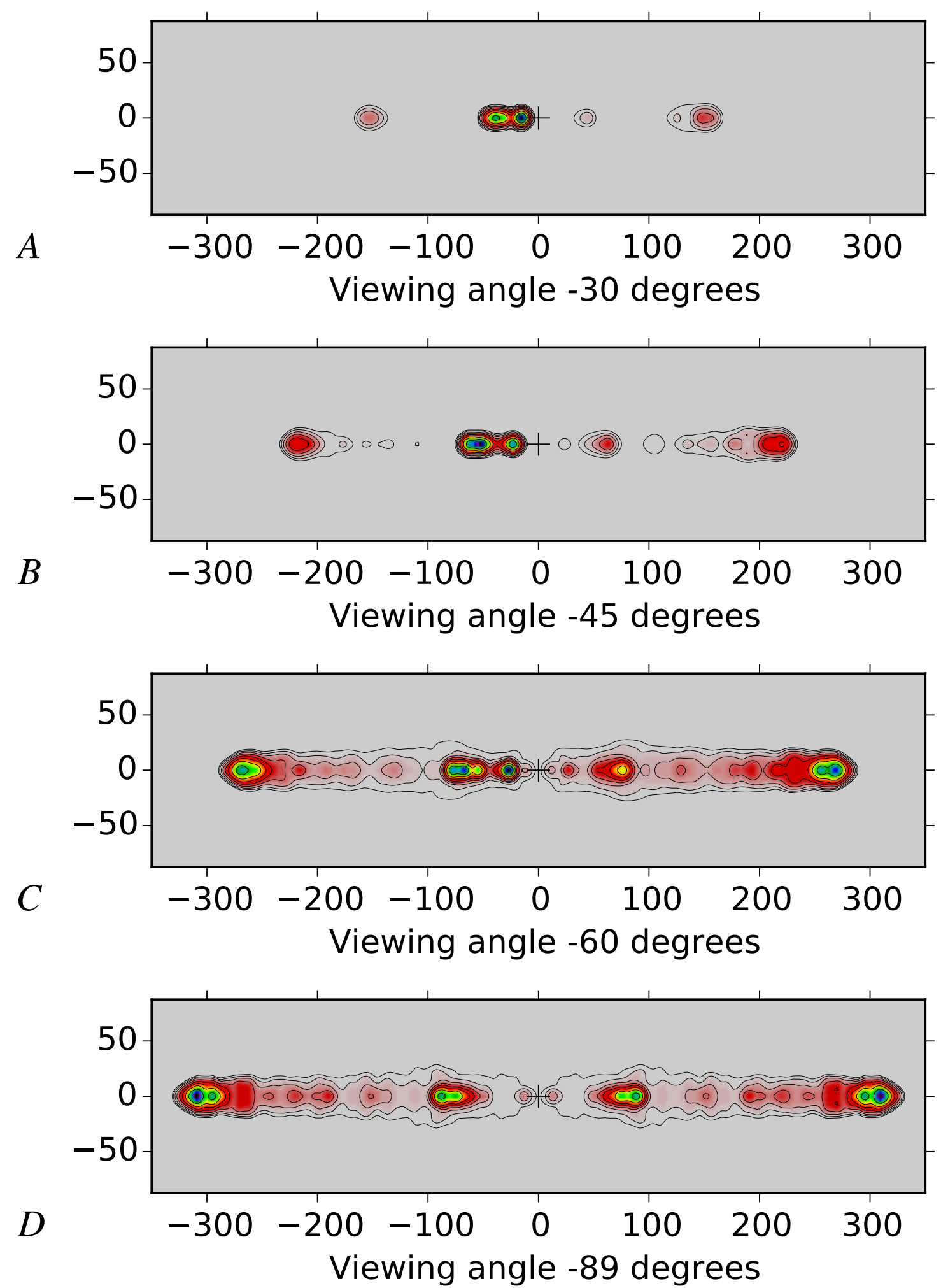

Figure 7. Influence of viewing angle, Doppler factor in the case of purely entangled magnetic fields (so $\Lambda=1.0$ ). Viewing angles from Panel A to D: $-30^{\circ},-45^{\circ},-60^{\circ},-89^{\circ}$. In this convention, a viewing angle between -90 degrees and 0 degrees correspond to the lower part of the jet pointing towards the observer. For 0 degrees, the jets are aligned with the line of sight and for 90 degrees, the jets are perpendicular to the line of sight. The flux levels are $I_{v} \in\{1,2,4,8,16,32,64,256\} \times I_{v, \min }$, with $I_{v, \min }=I_{v, \max } / 256$ and $I_{v, \max }=1$. 

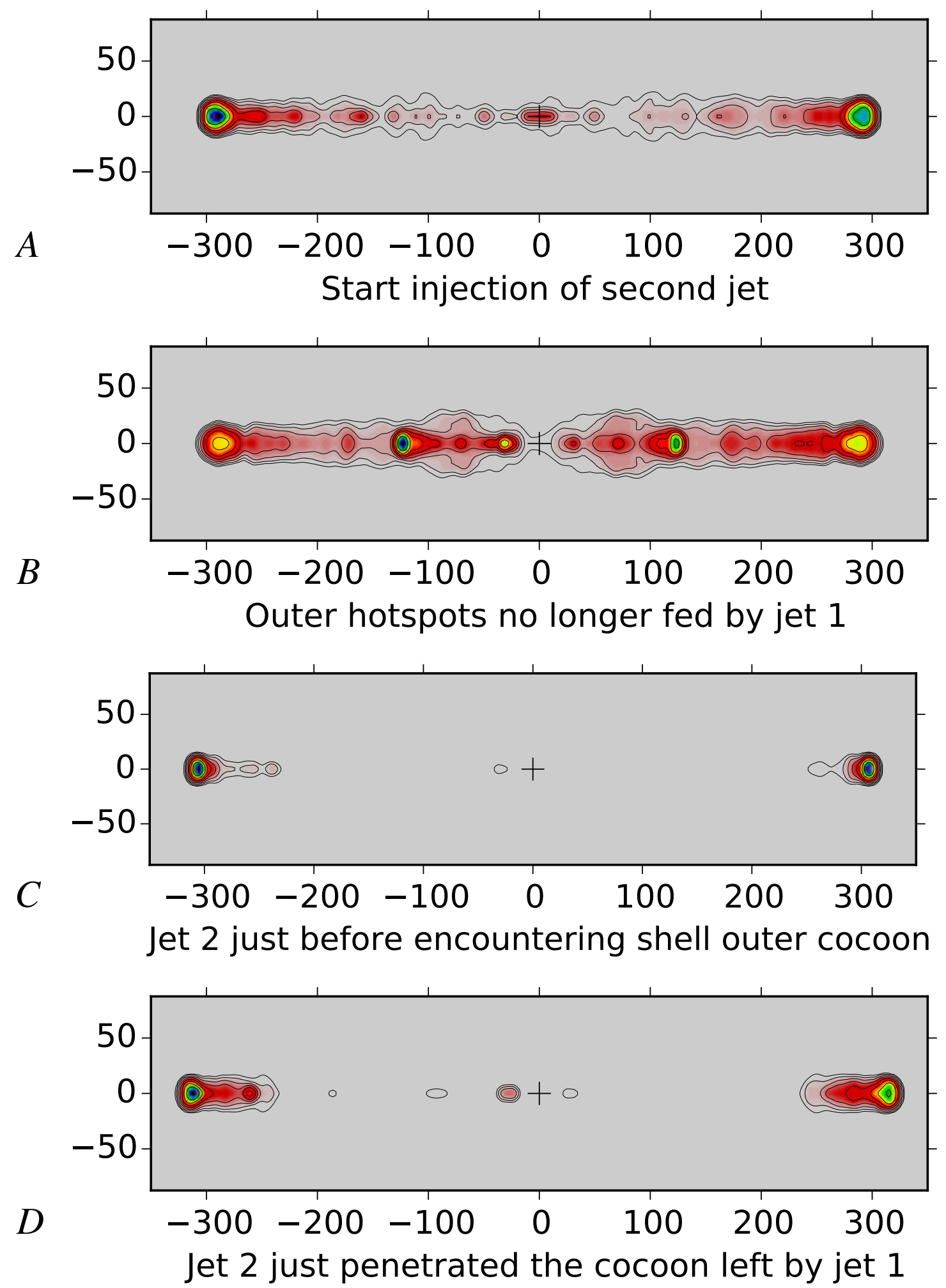

Figure 8. The isochoric jet model A2 shown at various stages during the evolution. Panel A: first jet at 16.0 Myr, just before the second jet is turned on. Panel B: $16.65 \mathrm{Myr}$, shortly after the outer hotspots are no longer fed by the first jets. Panel C: second jets at 18.0 Myr, just before the second jets will encounter the shell of the outer cocoon. Panel D: second jets at $18.7 \mathrm{Myr}$, shortly after the second jets have broken out of the outer cocoon. The flux levels are $I_{v} \in\{1,2,4,8,16,32,64,256\} \times I_{v, \min }$, with $I_{v, \min }=I_{v, \max } / 256$ and $I_{v, \max }=1$. 


\subsection{Differential light travel times}

The synthesized maps shown here do not take account of (differential) light travel time effects between the source and a distant observer, which affect the appearance of the source for such an observer. When the jets from a radio galaxy have a viewing angle $\vartheta \neq 90^{\circ}$, the distance from the observer to the advancing jet-head $\left(L_{\mathrm{a}}\right)$ is less then the distance from the observer to the receding jethead $\left(L_{\mathrm{r}}\right)$. The distance from Earth to an extra-galactic radio galaxy $\left(D_{\mathrm{s}}\right)$ is always much larger than the jet length $\left(D_{\mathrm{jt}}\right)$. Then the difference in light travel time to the observer for the advancing and the receding jet-head equals:

$\Delta t \propto \frac{L_{\mathrm{r}}-L_{\mathrm{a}}}{c} \approx \frac{2 D_{\mathrm{jt}} \cos \vartheta}{c}$,

where we have assumed that the advancing jet and the receding jet have equal length. This means that at a given moment of observation the recorded image of the jets becomes "progressively younger" if one moves from the receding hotspot towards the approaching hotspot. This is not included in our model. When viewing the synthesized synchrotron maps in this paper, one should bare in mind that the receding jet will appear younger (and therefore shorter) in reality. Almost all the synthesized synchrotron maps that we show assume a viewing angle of $\vartheta=-71^{\circ}$. The distance from the outer jet-heads to the AGN have an approximate size of $D_{\mathrm{jt}_{1}} \approx 300 \mathrm{kpc}$, while for the inner jet-heads we find $D_{\mathrm{jt}_{2}} \approx 90 \mathrm{kpc}$. Then the maximum time difference $(\Delta t)$ per age of the AGN $\left(t_{\mathrm{s}}\right)$ for the outer- and inner jet-heads respectively equals:

$$
\begin{aligned}
& \frac{\Delta t}{t_{\mathrm{s}}} \approx \frac{2 \cos \left(-71^{\circ}\right) D_{\mathrm{jt}_{1}}}{c t_{\mathrm{s}_{1}}} \approx 4 \text { per cent }, \\
& \frac{\Delta t}{t_{\mathrm{s}}} \approx \frac{2 \cos \left(-71^{\circ}\right) D_{\mathrm{jt}_{2}}}{c t_{\mathrm{s}_{2}}} \approx 32 \text { per cent },
\end{aligned}
$$

so that our neglect of the differential light travel effects for the inner jets should in reality be improved upon. We refer the reader to SW2, Fig. 3 for detailed information on the position of the jet-head of the first and the second jet, corresponding to the jet-length $D_{\mathrm{jt}_{1}}$ and $D_{\mathrm{jt}_{2}}$.

\section{CONCLUSIONS}

In this paper we have used the simulations of three different jet models in order to synthesize synchrotron radiation images at radio frequencies for double-double radio galaxies (DDRGs). As a reference source, we have chosen the DDRG J1835+6204 to compare our results with. The jet models are the homogeneous jet $H 2$; the isothermal jet $I 2$; and the (piecewise) isochoric jet $A 2$. In order to calculate the emissivity at frequency $v$, we calculated the Doppler factor; the (space-time dependent part of the) relativistic particle distribution $\mathcal{N}$; and the magnitude of the magnetic field component perpendicular to the line of sight $B_{\perp}$. We performed pure hydrodynamical simulations, and therefore approximated the (ordered, entangled, or mixed) magnetic fields. While this clearly is an approximation, it allowed us to explore the effect of field line geometry on the radio images. In this work we have studied the effects on the brightness contrasts of the synthesized synchrotron images of:

1 the radial profiles of the three jet models $H 2, I 2$ and $A 2$;

2 the various magnetic field configurations;

3 spectral ageing from synchrotron cooling;

4 contributions from jet components $\left(\theta_{\mathrm{sp}_{1}}, \theta_{\mathrm{sh}_{1}}, \theta_{\mathrm{sp}_{2}}, \theta_{\mathrm{sh}_{2}}\right)$;

5 the viewing angle of the jets;

6 the various epochs (phases) of the evolution of a DDRG.
We find the following conclusions:

(i) In all cases (where entangled magnetic fields are involved) the synthesized synchrotron images show two clear (pairs of) hotspots at $8 \mathrm{kpc}$ resolution (corresponding to a VLA radio observation at $4.8 \mathrm{GHz}$, and average beam size of 1.4"): The outer hotspots from the first (initial) jets, NW1 and SE1, and the inner hotspots from the second (restarted) jets, NW2 and SE2, and therefore satisfying the condition for a DDRG;

(ii) In all cases the expected effects of Doppler boosting/dimming are confirmed: we find that for jets that are approaching the observer, the associated hotspots and other radio features along its jet axis are Doppler boosted (brightened), whereas for the jets that are receding, these same radio features are Doppler deboosted (dimmed). Notably, the opposite behaviour in the back-flowing material can also clearly be seen;

(iii) For the observed DDRG J1835+6204 at $8 \mathrm{kpc}$ resolution and $4.8 \mathrm{GHz}$, we find the closest resemblance to the observations with the (piecewise) isochoric jet model $A 2$. That model generates synthesized synchrotron images consistent with $\mathrm{J} 1835+6204$, due to its relatively low radial jet integrity;

(iv) We find that the synchrotron radiation that is generated by the ordered magnetic fields appears as a small number of knots along the jet axis, in the direct vicinity of internal shocks, or just before the Mach disc of the jet-heads. When assuming a mix of entangled magnetic fields and ordered magnetic fields, we notice a shift in brightness contrasts. When the magnetic pressure of the ordered fields become significant ( $~ 250$ per cent of the total magnetic pressure), details of the cocoon start to fade in the synthesized images. We get the best resemblance with the observation of J1835+6204 when the magnetic pressure of the ordered fields is small compared to the total magnetic pressure $(\lesssim 30$ per cent, so $\Lambda \gtrsim 0.7)$;

(v) The effect of spectral ageing by synchrotron cooling becomes apparent at observation frequencies $v_{\mathrm{obs}} / v_{\infty, 0} \gtrsim 10^{-6}$, and becomes significant at frequencies $v_{\mathrm{obs}} / v_{\infty, 0} \gtrsim 10^{-3}$, where $v_{\infty, 0} \equiv 1$ denotes the cut-off frequency of the relativistic particle population at jet inlet. For $\mathrm{J} 1835+6204$ this cut-off frequency is approximately $v_{\infty} \approx$ $3.1 \cdot 10^{5} \mathrm{MHz}$. The most notable effect of spectral ageing in the synthesized synchrotron images is the fading of the cocoon. At relatively very high observation frequencies $v_{\mathrm{obs}} / v_{\infty, 0} \gtrsim 10^{-1}$ the effects of spectral ageing becomes so strong that also the outer hotspots of the jets NW1 and SE1 will vanish in the synchrotron maps. Taking the cut-off frequency for $\mathrm{J} 1835+6204$, this would then occur at an observation frequency of $v_{\mathrm{obs}} \sim 3 \cdot 10^{1} \mathrm{GHz}$.

(vi) We are able to separate the contributions in synchrotron radiation coming from the different jet components (the spine material, or the sheath material from the first jet, and equivalently for the second jet). We find that the first jets create a wide cocoon. The second jets create a very thin cocoon. The bow shock of disturbed material within the outer cocoon, which is pushed by the second jets is also quite bright, so that the inner hotspots also partially lightened up by the left-over material from the first jets. Finally we find that the contribution from jet spine material remains very close to the jet axis, while the contribution from the jet sheath material comes from both the jet and the surrounding cocoon.

(vii) The viewing angle has a strong effect on the observed size of the source, as well as the Doppler boosting/dimming of the jets. The Doppler effect is strongest for the inner jets, due to the fact that the jet-head advance speed of the inner jets $(\sim 0.7 \mathrm{c})$ is higher than that of the remaining outer jets $(\sim 0.045 \mathrm{c})$. When the viewing angle $\vartheta \sim-30^{\circ}$, the approaching inner jet is brightest, while the receding inner jet has virtually faded completely. Also the outer jets have 
almost faded completely. For even smaller viewing angles all that remains in the synthesized image will be the approaching inner jet: A DDRG structure would no longer be recognisable.

(viii) The time it takes for the first jet to fade after the central engine has turned off is small compared to the life time of the radio source. Therefore, the chance of detecting an episodic AGN jet radio source as an actual DDRG is small: the second jets must be turned on again well before the first jets have completely faded. Within a few hundred thousand years the radio morphology of the source would look completely different. We find that when the restarted jets start to penetrate the cocoon that was left behind by the first jets nearly all that is visible are the termination shocks at the jet-heads. It is at this point that a strong back flow begins to form again, and more of the cocoon structure will arise again.

\subsection{Outlook}

From the results we've been able to derive contributions from magnetic field components for the entangled, the azimuthal, the poloidal and the helical, and the mixed magnetic field configurations. In order to do so, we had to make the necessary approximations and assumptions. Adding actual magnetic fields to this model (switch to a full MHD module) would be a valuable contribution. Such SRMHD simulations would also allow for a more self-consistent quantification of the measured polarization. A few more aspects are mentioned in this outlook, to be considered for future work.

\subsubsection{Inverse-Compton losses}

When discussing spectral ageing, we ignored the potential influence of Inverse-Compton losses, which is likely a significant contributor to radiative losses. Hardcastle (2018) discusses a semianalytical model for the evolution of powerful radio galaxies, where trends between jet power and luminosity are reproduced. Inverse Compton losses arise from scattering of the Cosmic Microwave Background, whose radiation field energy density is about $U_{\text {rad }} \approx$ $4.17 \times 10^{-13}(1+z)^{4} \mathrm{erg} / \mathrm{cm}^{3}$ in intergalactic space at redshift $z$. In practice, this would imply that our energy loss formulae must replace the magnetic field with an effective field:

$\frac{B_{\mathrm{eff}}^{2}}{8 \pi}=\frac{B^{2}}{8 \pi}+U_{\mathrm{rad}}$.

At the same time, the synchrotron emission must still employ the actual field pressure $B^{2} / 8 \pi$. Since this in practice introduces yet another degree of freedom when generating radio maps from our SRHD simulations, we did not incorporate this effect here. In future SRMHD runs, where the $B^{2} / 8 \pi$ is self-consistently computed, these losses should be considered too.

\subsubsection{Dimensionality of the simulations}

These simulations have been performed in a 2.5D (cylindrical symmetric) setting. Many observed features give a good notion on the processes at hand. However, in a full 3D setting the jet flow, as well as the back-flowing material are able to effectively propagate in one extra dimension. This causes a less symmetrical flow and allows for more instabilities to arise. For example, eddies (vortices) that form at the jet-head in the back-flowing material in the $2.5 \mathrm{D}$ case all have approximately the same size and show similar flowing behaviour. They propagate downwards relatively steady along the jet-axis, away from the jet-head. In a 3D case, these eddies will most likely have different shapes and not form with the same degree of symmetry as that imposed in a $2.5 \mathrm{D}$ setting. Moreover, it is to be expected that the jet axis itself will begin to wobble under the influences of instabilities, instead of being a perfectly straight line. This will inevitably have an effect on the jet-cocoon interaction, the related jet pinches and internal shocks, which in turn all have an effect on the related observed radio features. Therefore, the results should be interpreted with some caution. The advantage of performing $2.5 \mathrm{D}$ simulations compared to $3 \mathrm{D}$ simulations is that we are able to resolve radio structures in much more detail, while being able to let the jets evolve on a very large scale.

\subsubsection{Renewed injection of non-thermal particles}

In this work we have not involved the injection of a fresh nonthermal particle population at internal shocks, or at the termination shock of the jets. If these fresh injections were to be implemented, the brightness contrasts within the synthesized images would of course change. The effect of injecting fresh relativistic particles will, however, become most apparent at those frequencies were spectral ageing has already played a significant role. A realistic implementation of the injection of fresh populations of relativistic particles would require an observational study of particle populations near internal shocks and the termination shock.

\section{ACKNOWLEDGEMENTS}

This research is funded by the Nederlandse Onderzoekschool Voor Astronomie (NOVA). RK acknowledges a joint FWO-NSFC grant G0E9619N and from Internal Funds KU Leuven, project C14/19/089 TRACESpace. We thank the anonymous referee for useful feedback and suggestions that improved our manuscript.

\section{DATA AVAILABILITY}

The data underlying this article will be shared on reasonable request to the corresponding author.

\section{REFERENCES}

Achterberg A., Norman C. A., 2018, MNRAS, 479, 1747

Aloy M.-A., Gómez J.-L., Ibáñez J.-M., Martí J.-M., Müller E., 2000, ApJ, 528, L85

Begelman M. C., Blandford R. D., Rees M. J., 1984, Reviews of Modern Physics, 56, 255

Bird J., Martini P., Kaiser C., 2008, ApJ, 676, 147

Blandford R., Meier D., Readhead A., 2019, ARA\&A, 57, 467

Blandford R. D., Payne D. G., 1982, MNRAS, 199, 883

Blandford R. D., Znajek R. L., 1977, MNRAS, 179, 433

Blumenthal G. R., Mathews W. G., 1976, ApJ, 203, 714

Bromberg O., Nakar E., Piran T., Sari R., 2011, ApJ, 740, 100

Callingham J. R., Ekers R. D., Gaensler B. M., et al. 2017, ApJ, 836, 174

Camus N. F., 2009, PhD thesis, The University of Leeds

Camus N. F., Komissarov S. S., Bucciantini N., Hughes P. A., 2009, MN-

RAS, 400, 1241

Chatterjee K., Liska M., Tchekhovskoy A., Markoff S. B., 2019, MNRAS, 490, 2200

Chon G., Böhringer H., Krause M., Trümper J., 2012, A\&A, 545, L3

Clarke D. A., Burns J. O., 1991, ApJ, 369, 308 
Croston J. H., Hardcastle M. J., Harris D. E., Belsole E., Birkinshaw M., Worrall D. M., 2005, ApJ, 626, 733

Croston J. H., Ineson J., Hardcastle M. J., 2018, MNRAS, 476, 1614

Dabhade P., Gaikwad M., Bagchi J., Pand ey-Pommier M., Sankhyayan S., Raychaudhury S., 2017, MNRAS, 469, 2886

Del Zanna L., Volpi D., Amato E., Bucciantini N., 2006, A\&A, 453, 621

Fuentes A., Gómez J. L., Martí J. M., Perucho M., 2018, ApJ, 860, 121

Gabuzda D. C., Knuettel S., Reardon B., 2015, Monthly Notices of the

Royal Astronomical Society, 450, 24412450

Ghisellini G., Tavecchio F., Chiaberge M., 2005, A\&A, 432, 401

Giroletti M., Giovannini G., Feretti L., et al. 2004, ApJ, 600, 127

Goedbloed J. P., Keppens R., Poedts S., 2019, Magnetohydrodynamics of Laboratory and Astrophysical Plasmas. Cambridge U. Press, Cambridge

Gómez J. L., Agudo I., Marscher A. P., Jorstad S. G., Roca-Sogorb M., 2008, Mem. Soc. Astron. Italiana, 79, 1157

Hardcastle M. J., 2018, MNRAS, 475, 2768

Hardcastle M. J., Williams W. L., Best P. N., Croston J. H., et al. 2019, A\&A, 622, A12

Homan D. C., Kovalev Y. Y., Lister M. L., et al. 2006, ApJ, 642, L115

Kaiser C. R., Schoenmakers A. P., Röttgering H. J. A., 2000, MNRAS, 315,381

Kawakatu N., Kino M., Takahara F., 2016, MNRAS, 457, 1124

Kellermann K. I., Kovalev Y. Y., Lister M. L., Homan D. C., et al. 2007, Ap\&SS, 311, 231

Keppens R., Meliani Z., van der Holst B., Casse F., 2008, A\&A, 486, 663

Keppens R., Meliani Z., van Marle A. J., Delmont P., Vlasis A., van der Holst B., 2012, Journal of Computational Physics, 231, 718

Konar C., Hardcastle M. J., 2013, MNRAS, 436, 1595

Konar C., Hardcastle M. J., Jamrozy M., Croston J. H., 2013, MNRAS, 430, 2137

Konar C., Hardcastle M. J., Jamrozy M., Croston J. H., Nandi S., 2012, MNRAS, 424, 1061

Koren B., 1993, A robust upwind discretization method for advection, diffusion and source terms, in: C.B. Vreugdenhil, B. Koren (Eds.), Numerical Methods for AdvectionDiffusion Problems, Notes on Numerical Fluid Mechanics, vol 45, Vieweg, Braunschweig, 1993, p117

Kuźmicz A., Jamrozy M., Kozieł-Wierzbowska D., Weżgowiec M., 2017,

Monthly Notices of the Royal Astronomical Society, 471, 3806

Laing R. A., Bridle A. H., 2014, MNRAS, 437, 3405

Lee S.-J., Chattopadhyay I., Kumar R., et al. 2016, ApJ, 831, 33

Longair M. S., 2011, High Energy Astrophysics (3d Edition)

Mahatma V. H., Hardcastle M. J., Croston J. H., Harwood J., Ineson J., Moldon J., 2020, MNRAS, 491, 5015

Mahatma V. H., Hardcastle M. J., Williams W. L., et al. 2019, Astronomy and Astrophysics, 622, A13

Marecki A., Jamrozy M., Machalski J., 2016, MNRAS, 463, 338

Martí J.-M., 2019, Galaxies, 7, 24

McKinney J. C., Blandford R. D., 2009, MNRAS, 394, L126

Mendygral P. J., Jones T. W., Dolag K., 2012, ApJ, 750, 166

Mignone A., Bodo G., 2005, MNRAS, 364, 126

Nandi S., Jamrozy M., Roy R., Larsson J., Saikia D. J., Baes M., Singh M., 2017, MNRAS, 467, L56

Nandi S., Saikia D. J., Roy R., Dabhade P., et al. 2019, Monthly Notices of the Royal Astronomical Society, 486, 5158

Orrù E., van Velzen S., Pizzo R. F., Yatawatta S., et al. 2015, A\&A, 584, A112

Park J., Hada K., Kino M., Nakamura Masanori e. a., 2019, ApJ, 887, 147

Porth O., Chatterjee K., Narayan R., Gammie C. F., et al. 2019, The Astrophysical Journal Supplement Series, 243, 26

Porth O., Komissarov S. S., Keppens R., 2014, MNRAS, 438, 278

Porth O., Xia C., Hendrix T., Moschou S. P., Keppens R., 2014, ApJS, 214, 4

Prior C., Gourgouliatos K., 2019, A\&A, 622, A122

Refaelovich M., Soker N., 2012, ApJ, 755, L3

Rybicki G. B., Lightman A. P., 1986, Radiative Processes in Astrophysics Safouris V., Subrahmanyan R., Bicknell G. V., Saripalli L., 2008, MNRAS, 385, 2117
Saikia D. J., Jamrozy M., 2009, Bulletin of the Astronomical Society of India, 37, 63

Schoenmakers A. P., de Bruyn A. G., Röttgering H. J. A., van der Laan H., 2000, MNRAS, 315, 395

Schoenmakers A. P., de Bruyn A. G., Röttgering H. J. A., van der Laan

H., Kaiser C. R., 2000, MNRAS, 315, 371

Shimwell T. W., Tasse C., Hardcastle M. J., Mechev A. P., et al. 2019, A\&A, 622, A1

Sol H., Pelletier G., Asseo E., 1989, MNRAS, 237, 411

Stepanovs D., Fendt C., Sheikhnezami S., 2014, ApJ, 796, 29

Synge J. L., 1957, The Relativistic Gas. North-Holland Pub. Co. (Amsterdam and New York)

Tchekhovskoy A., Narayan R., McKinney J. C., 2011, Monthly Notices of the Royal Astronomical Society: Letters, 418, L79L83

Teunissen J., Keppens R., 2019, arXiv e-prints, p. arXiv:1901.11370

Toro E. F., Spruce M., Speares W., 1994, Shock Waves, 4, 25

Turner R. J., Shabala S. S., 2015, ApJ, 806, 59

Walg S., Achterberg A., Markoff S., Keppens R., Meliani Z., 2013, MNRAS, 433, 1453

Walg S., Achterberg A., Markoff S., Keppens R., Porth O., 2014, MNRAS, 439, 3969

Webb G. M., 1985, ApJ, 296, 319

Wilson M. J., 1984, MNRAS, 209, 923

Xia C., Teunissen J., El Mellah I., Chané E., Keppens R., 2018, ApJS, 234, 30

Zamaninasab M., Clausen-Brown E., Savolainen T., Tchekhovskoy A., 2014, Nature, 510, 126

This paper has been typeset from a $\mathrm{T}_{\mathrm{E}} \mathrm{X} / \mathrm{L}_{\mathrm{E}} \mathrm{X}$ file prepared by the author.

\section{APPENDIX A:}

\section{A1 Advecting a populations of relativistic leptons: adiabatic} limit

In this Appendix we briefly consider the transport equation for relativistic leptons (electrons and/or positrons) in a relativistic flow. The basic assumption is that leptons are advected passively, with negligible diffusion with respect to the flow. This implies strong collisional coupling between the bulk flow and the population of leptons, mediated by scattering due to low-frequency MHD waves. In this situation the leptons change their energy: they lose energy due to radiation losses (mostly synchrotron losses) and expansion losses, and can gain energy if the flow is compressed. Notationwise we follow the main paper: $\epsilon^{\prime}=\sqrt{\left|\boldsymbol{p}^{\prime}\right|^{2}+m_{\mathrm{e}}^{2}}$ is the particle energy in the local fluid rest frame (FRF) (i.e. comoving energy), in units where $c=1$ (which we will assume in the appendices from this point on), and where $m_{\mathrm{e}}$ is the electron rest-mass. Quite generally we will employ primes to denote quantities measured in the local fluid rest frame. We employ (following Achterberg \& Norman 2018) mixed phase-space variables, where particle momentum is measured in the FRF, but space-time position $x^{\mu}=(\boldsymbol{r}, t)$ is measured in the laboratory frame (observer's frame). Neglecting the effects of diffusion with respect to the flow and viscous heating of the leptons the proper particle number density per unit comoving energy in mixed variables,

$\mathcal{N}_{\epsilon^{\prime}}(\boldsymbol{r}, t)=\mathcal{N}\left(\boldsymbol{r}, t, \epsilon^{\prime}\right) \equiv \frac{\mathrm{d} n^{\prime}}{\mathrm{d} \epsilon^{\prime}}$

satisfies, neglecting radiation losses for now as well as the possible injection of fresh leptons,

$\frac{\partial}{\partial x^{\mu}}\left(U^{\mu} \mathcal{N}_{\epsilon^{\prime}}\right)-\frac{\partial}{\partial \epsilon^{\prime}}\left[\frac{\epsilon^{\prime}}{3}(\nabla \cdot U) \mathcal{N}_{\epsilon^{\prime}}\right]=0$. 
We employ a covariant notation for the four-divergence of the plasma flow:

$\nabla \cdot U \equiv \frac{\partial U^{\mu}}{\partial x^{\mu}}=\frac{\partial \gamma}{\partial t}+\nabla \cdot \boldsymbol{U}$

with four-velocity $U^{\mu}=(\gamma, \boldsymbol{U})$ where $\boldsymbol{U}=\gamma \boldsymbol{V}$ and $\gamma=1 / \sqrt{1-|\boldsymbol{V}|^{2}}$ the bulk flow Lorentz factor. The second term on the left-hand side of (A2) describes the energy change due to expansion losses (or compression gains), c.f. Webb (1985) and Achterberg \& Norman (2018), Eqn. 8.95:

$\frac{\mathrm{d} \epsilon^{\prime}}{\mathrm{d} \tau}=-\frac{\epsilon^{\prime}}{3}(\nabla \cdot U)=-\frac{\epsilon^{\prime}}{3}\left(\frac{\partial \gamma}{\partial t}+\nabla \cdot U\right)$.

Here

$\frac{\mathrm{d}}{\mathrm{d} \tau}=U^{\mu} \frac{\partial}{\partial x^{\mu}}=\gamma\left(\frac{\partial}{\partial t}+(\boldsymbol{V} \cdot \boldsymbol{\nabla})\right) \equiv \gamma \frac{\mathrm{d}}{\mathrm{d} t}$

is the covariant comoving derivative in the bulk flow. Mass conservation in the bulk flow with lab frame density $\rho=\gamma \rho^{\prime}$ reads

$\frac{\partial \rho}{\partial t}+\boldsymbol{\nabla} \cdot(\rho \boldsymbol{V})=\frac{\partial\left(\gamma \rho^{\prime}\right)}{\partial t}+\boldsymbol{\nabla} \cdot\left(\rho^{\prime} \boldsymbol{U}\right)=0$,

or equivalently

$\frac{1}{\rho^{\prime}} \frac{\mathrm{d} \rho^{\prime}}{\mathrm{d} t}=-\left(\frac{1}{\gamma} \frac{\mathrm{d} \gamma}{\mathrm{d} t}+\boldsymbol{\nabla} \cdot \boldsymbol{V}\right)$.

For a power-law distribution in comoving energy of the form

$\mathcal{N}_{\epsilon^{\prime}}=\mathcal{N}(\boldsymbol{r}, t)\left(\epsilon^{\prime}\right)^{-s}$,

the transport equation can be written as

$\gamma \frac{\mathrm{d} \mathcal{N}}{\mathrm{d} t}+\left(\frac{s+2}{3}\right)\left(\frac{\partial \gamma}{\partial t}+\boldsymbol{\nabla} \cdot \boldsymbol{U}\right) \mathcal{N}=0$.

Then Eqn. A7 for the proper plasma density $\rho^{\prime}$ implies:

$\mathcal{N}=\mathcal{N}_{0}\left(\frac{\rho^{\prime}}{\rho_{0}^{\prime}}\right)^{(s+2) / 3} \propto\left(\rho^{\prime}\right)^{(s+2) / 3}$,

with $\mathcal{N}_{0}(\boldsymbol{r}, t)$ and $\rho^{\prime}{ }_{0}(\boldsymbol{r}, t)$ corresponding to the values at position $\boldsymbol{r}_{0}$ at time $t_{0}$. This is a good approximation for those energies well below the energy where the synchrotron break in the spectrum occurs and synchrotron losses need to be taken into account.

For ultra-relativistic particles the energy density in the plasma restframe, which we denote simply by $\mathcal{W}$, equals

$\mathcal{W}(\boldsymbol{r}, t)=3 P(\boldsymbol{r}, t)=\int_{0}^{\infty} \mathrm{d} \epsilon^{\prime} \epsilon^{\prime} \mathcal{N}\left(\boldsymbol{r}, t, \epsilon^{\prime}\right)$.

Here $P(\boldsymbol{r}, t)$ is the pressure. Multiplying transport equation A2 by $\epsilon^{\prime}$ and then integrating over plasma rest-frame particle energy $\epsilon^{\prime}$ yields, after a partial integration:

$\frac{\partial}{\partial x^{\mu}}\left(U^{\mu} \mathcal{W}\right)+\frac{(\nabla \cdot U)}{3} \mathcal{W}=0$.

This can be written as

$\gamma \frac{\mathrm{d} \mathcal{W}}{\mathrm{d} t}+\frac{4}{3}\left(\frac{\partial \gamma}{\partial t}+\boldsymbol{\nabla} \cdot \boldsymbol{U}\right) \boldsymbol{W}=0$

or equivalently

$\frac{\mathrm{d} P}{\mathrm{~d} \tau}=-\frac{4}{3} P(\nabla \cdot U)$,

With density relation (A7) this implies that

$\mathcal{W}, P \propto\left(\rho^{\prime}\right)^{4 / 3}$,

the usual relation for an ideal, adiabatic and relativistically hot gas.

\section{A2 The $\mathcal{N}$-Tracer model}

In the simulations presented in the main paper we assume that each jet component (i.e. jet spine 1 , jet sheath $1, \ldots$ ) has its own population of relativistic particles. Each jet component ' $A$ ' is given a separate tracer $\theta_{\mathrm{A}}^{\prime}(\boldsymbol{r}, t)$ (also measured in the plasma rest-frame). In the numerical simulations that are used in this paper, the tracer values of the individual jet component in a certain grid cell $\boldsymbol{r}_{i}$ at a time $t$ represents the mass fraction of that jet component (see SW1 or SW2). In other words, the mass density of jet component $A$ is calculated according to $\rho_{\mathrm{A}}^{\prime}(\boldsymbol{r}, t)=\theta_{\mathrm{A}}^{\prime}(\boldsymbol{r}, t) \rho^{\prime}(\boldsymbol{r}, t)$. In particular, $\theta_{\mathrm{A}}^{\prime}=0$ in a certain grid cell means that jet component $A$ is absent in that grid cell, whereas $\theta_{\mathrm{A}}^{\prime}=1$ means that the material inside the cell purely consists of jet component $A$. Values in between $0<\theta_{\mathrm{A}}^{\prime}<1$ indicate that the grid cell consists of multiple components. Therefore it is reasonable to assume that the relativistic particle distribution $\mathcal{N}_{A}$, for a jet component $A$, with mass fraction (or tracer value) $\theta_{A}^{\prime}$ in a grid cell $\boldsymbol{r}$ at time $t$ can be scaled with the bulk mass density $\rho$ according to:

$\mathcal{N}_{\mathrm{A}} \propto \mathcal{N}_{\mathrm{A} 0}\left(\frac{\rho_{\mathrm{A}}^{\prime}}{\rho_{\mathrm{A} 0}^{\prime}}\right)^{\frac{s+2}{3}}=\mathcal{N}_{\mathrm{A} 0}\left(\frac{\theta_{\mathrm{A}}^{\prime} \rho^{\prime}}{\rho_{\mathrm{A} 0}^{\prime}}\right)^{\frac{s+2}{3}}$,

neglecting the effects of synchrotron cooling, and with $\mathcal{N}_{\mathrm{A} 0}(\boldsymbol{r}, t)$ and $\rho_{\mathrm{A} 0}(\boldsymbol{r}, t)$ the number density and mass density of the relativistic particles of jet component $A$ at the injection site (the jet inlet). The total synchrotron emissivity as a result of all the jet components $A$ is the sum of the synchrotron emissivities of the individual jet components. In this way, we finally end up with:

$j_{v}=\Sigma_{\mathrm{A}} j_{v, \mathrm{~A}} \propto \Sigma_{\mathrm{A}} \mathcal{D}^{2+\alpha} \mathcal{N}_{\mathrm{A}}\left(B_{\perp}^{\prime}\right)^{\alpha+1}(v)^{-\alpha}$.

Here $\alpha$ is the usual spectral spectral index of optically thin synchrotron radiation, related to the slope $s$ of the electron distribution by $\alpha=(s-1) / 2$, c.f. Rybicki \& Lightman (1986).

\section{A3 The N-Cooling model}

In the previous section we calculated the distribution $\mathcal{N}(\boldsymbol{r}, t)$ of the relativistic electrons for the case that synchrotron losses can be neglected. This distribution can also be derived for the case that synchrotron losses are included (for a full derivation, see Camus 2009. Section 4.3.4). In short the derivation goes as follows:

It is generally believed that electrons are accelerated when crossing (strong) shocks. Therefore, a shock is assumed to be the injection site of a relativistic particle population. After crossing the shock, the energy evolution of a single particle moving along stream lines (of the post-shock plasma) can be written as:

$\frac{\mathrm{d}}{\mathrm{d} t}\left\{\ln \left(\epsilon^{\prime}\right)\right\}=\frac{\mathrm{d}}{\mathrm{d} t}\left\{\ln \left[\left(n^{\prime}\right)^{\frac{1}{3}}\right]\right\}+\frac{1}{\epsilon^{\prime}}\left(\frac{\mathrm{d} \epsilon_{\mathrm{sy}}^{\prime}}{\mathrm{d} t}\right)$,

the sum of adiabatic losses (the first term on the right hand side) and radiative energy losses (the second term), and $n^{\prime}(\boldsymbol{r}, t)$ is the proper number density of the relativistic leptons, defined as

$n^{\prime}(\boldsymbol{r}, t)=\int_{0}^{\infty} \mathrm{d} \epsilon^{\prime} \mathcal{N}_{\epsilon^{\prime}}(\boldsymbol{r}, t)$.

The adiabatic loss term uses that $n^{\prime}(\boldsymbol{r}, t)$ satisfies (away from shocks)

$\frac{\partial}{\partial x^{\mu}}\left(U^{\mu} n^{\prime}(\boldsymbol{r}, t)\right)=0$,

which follows straightforwardly from an integration over comoving energy $\epsilon^{\prime}$ of Eqn. A2, This equation, together with Eqn. (A4) for the adiabatic energy loss rate, implies 
$\left(\frac{1}{\epsilon^{\prime}} \frac{\mathrm{d} \epsilon^{\prime}}{\mathrm{d} \tau}\right)_{\mathrm{ad}}=-\frac{1}{3}(\boldsymbol{\nabla} \cdot U)=\frac{1}{3}\left(\frac{1}{n^{\prime}} \frac{\mathrm{d} n^{\prime}}{\mathrm{d} \tau}\right)$.

When the relativistic electron moves in a local FRF magnetic field $B^{\prime}$, the energy loss due to synchrotron emission is:

$\left(\frac{\mathrm{d} \epsilon^{\prime}}{\mathrm{d} t}\right)_{\mathrm{sy}}=-\beta_{\mathrm{sy}}\left(B^{\prime}\right)^{2}\left(\epsilon^{\prime}\right)^{2}$,

where $\beta_{\mathrm{sy}}=2 e^{4} / 3 m_{\mathrm{e}}^{2}$ with $e$ the unit of electric charge and $m_{\mathrm{e}}$ the electron mass, using $c=1$ as before.

Substituting A22 into A18, one can rewrite A18 as:

$\frac{\mathrm{d}}{\mathrm{d} t}\left\{\ln \left[\frac{\epsilon^{\prime}}{\left(n_{\mathrm{e}}^{\prime}\right)^{1 / 3}}\right]\right\}=-\beta_{\mathrm{sy}}\left(B^{\prime}\right)^{2}\left(n^{\prime}\right)^{1 / 3}\left[\frac{\epsilon^{\prime}}{\left(n^{\prime}\right)^{1 / 3}}\right]$.

The solution, obtained by time-integration, is

$\frac{1}{\tilde{\epsilon}^{\prime}(t)}-\frac{1}{\epsilon^{\prime} \tilde{\left.t_{0}\right)}}=\int_{t_{0}}^{t} \mathrm{~d} t^{\prime} \beta_{\text {sy }}\left(B^{\prime}\right)^{2}\left(n^{\prime}\right)^{1 / 3} \equiv \frac{1}{\tilde{\epsilon_{\infty}^{\prime}(t)}}$.

Here define $\tilde{\epsilon}^{\prime}(\boldsymbol{r}, t) \equiv \epsilon^{\prime}(\boldsymbol{r}, t) /\left[n^{\prime}(\boldsymbol{r}, t)\right]^{1 / 3}$. Also, $t_{0}$ corresponds to the time of injection of the particles at the jet inlet. Relation A24 defines the quantity $\epsilon_{\infty}^{\prime}(t)=\left[n^{\prime}(t)\right]^{1 / 3} \tilde{\epsilon}_{\infty}^{\prime}(t)$, the energy where synchrotron losses modify the spectrum. Note that (by definition) $\epsilon_{\infty}^{\prime}=\infty$ at $t=t_{0}$. We can solve for the corresponding energy $\epsilon^{\prime}(t)$ (for $t \geqslant t_{0}$ ):

$\epsilon^{\prime}(t)=\frac{\epsilon_{0}^{\prime} \epsilon_{\infty}^{\prime}(t)\left[n^{\prime}(t)\right]^{1 / 3}}{\epsilon_{0}^{\prime}\left[n^{\prime}(t)\right]^{1 / 3}+\epsilon_{\infty}^{\prime}(t)\left[n_{0}^{\prime}\right]^{1 / 3}}$.

Here $n_{0}^{\prime}=n^{\prime}\left(\boldsymbol{r}_{0}, t_{0}\right)$ and $\epsilon_{0}^{\prime}=\epsilon^{\prime}\left(\boldsymbol{r}_{0}, t_{0}\right)$. Note that $\epsilon^{\prime}(t) \leqslant \epsilon_{0}^{\prime}$, as expected, when the jet expands so that $n^{\prime} \leqslant n_{0}^{\prime}$.

In this work, the relativistic particles are only injected at the jet inlet: no fresh relativistic particles are injected anywhere along the flow. Since the jet expands globally as one moves along the jet axis towards the jet-head, there will be mainly energy losses (except for local turbulence, or near shocks where, locally, $\boldsymbol{\nabla} \cdot \boldsymbol{V}<0$ ). This determines the energy distribution of the relativistic leptons. Let us define the normalized distribution function $f\left(\epsilon^{\prime}, \boldsymbol{r}, t\right)$ by

$f\left(\epsilon^{\prime}, \boldsymbol{r}, t\right)=\frac{\mathcal{N}_{\epsilon^{\prime}}(\boldsymbol{r}, t)}{n^{\prime}(\boldsymbol{r}, t)} \Longleftrightarrow \int_{0}^{\infty} \mathrm{d} \epsilon^{\prime} f\left(\epsilon^{\prime}, \boldsymbol{r}, t\right)=1$.

We assume $f\left(\epsilon^{\prime}, \boldsymbol{r}, t\right)$ to satisfy the following initial condition at injection (power-law injection):

$f\left(\epsilon_{0}^{\prime}, \boldsymbol{r}_{0}, t_{0}\right)=\kappa\left(\epsilon_{0}^{\prime}\right)^{-s}$,

with $\kappa$ some constant that ensures the proper normalization of $f\left(\epsilon_{0}^{\prime}, \boldsymbol{r}_{0}, t_{0}\right)$. Conservation of the number of particles, thereby preserving the unit normalization of $f\left(\epsilon^{\prime}, \boldsymbol{r}, t\right)$, leads to:

$f\left(\epsilon^{\prime}, \boldsymbol{r}, t\right)=f\left(\epsilon_{0}^{\prime}, \boldsymbol{r}_{0}, t_{0}\right)\left(\frac{\mathrm{d} \epsilon^{\prime}}{\mathrm{d} \epsilon_{0}^{\prime}}\right)^{-1}=f\left(\epsilon_{0}^{\prime}, \boldsymbol{r}_{0}, t_{0}\right)\left(\frac{\epsilon^{\prime}}{\epsilon_{0}^{\prime}}\right)^{-2}\left(\frac{n^{\prime}}{n_{0}^{\prime}}\right)^{1 / 3}$

The second equality follows straightforwardly from relation (A25) which yields $\mathrm{d} \epsilon^{\prime} / \mathrm{d} \epsilon_{0}^{\prime}=\left(\epsilon^{\prime} / \epsilon_{0}^{\prime}\right)^{2}\left(n^{\prime} / n_{0}^{\prime}\right)^{-1 / 3}$. Adopting A27) for $f\left(\epsilon_{0}^{\prime}, \boldsymbol{r}_{0}, t_{0}\right)$ we find:

$f\left(\epsilon^{\prime}, \boldsymbol{r}, t\right)=\kappa\left(\epsilon^{\prime}\right)^{-s}\left(\frac{\epsilon^{\prime}}{\epsilon_{0}^{\prime}}\right)^{s-2}\left(\frac{n^{\prime}}{n_{0}^{\prime}}\right)^{1 / 3}$.

Definition A26 then gives

$\mathcal{N}_{\epsilon^{\prime}}(\boldsymbol{r}, t)=n^{\prime} f\left(\epsilon^{\prime}, \boldsymbol{r}, t\right)=n_{0}^{\prime} \kappa\left(\epsilon^{\prime}\right)^{-s}\left(\frac{\epsilon^{\prime}}{\epsilon_{0}^{\prime}}\right)^{s-2}\left(\frac{n^{\prime}}{n_{0}^{\prime}}\right)^{4 / 3}$
The two factors involving $\epsilon^{\prime} / \epsilon_{0}^{\prime}$ and $n^{\prime} / n_{0}^{\prime}$ give how much the spectrum is depressed with respect to the original power law (as observations show that $s=2 \alpha+1>2$ for most optically thin synchrotron emission) by the combined action of expansion and energy losses. Using that relation $\mathrm{A} 25$ can be manipulated into

$\frac{\epsilon^{\prime}}{\epsilon_{0}^{\prime}}=\left(\frac{n^{\prime}}{n_{0}^{\prime}}\right)^{1 / 3}\left(1-\frac{\epsilon^{\prime}}{\epsilon_{\infty}^{\prime}}\right)$,

which incidentally shows that the spectrum must cut off at $\epsilon^{\prime}=\epsilon_{\infty}^{\prime}$, one can write $\mathcal{N}_{\epsilon^{\prime}}(\boldsymbol{r}, t)$ as

$\mathcal{N}_{\epsilon^{\prime}}(\boldsymbol{r}, t)=n_{0}^{\prime} \kappa\left(\epsilon^{\prime}\right)^{-s}\left(\frac{n^{\prime}}{n_{0}^{\prime}}\right)^{(s+2) / 3}\left(1-\frac{\epsilon^{\prime}}{\epsilon_{\infty}^{\prime}}\right)^{s-2}$.

\section{A4 Implementation in the numerical code}

In order to calculate $\mathcal{N}_{\epsilon^{\prime}}(\boldsymbol{r}, t)$, we need to determine the values of the relativistic proper number density $n^{\prime}(\boldsymbol{r}, t)$; its value at the time of injection $n_{\mathrm{e} 0}^{\prime}(\boldsymbol{r}, t)$; the particle energy $\epsilon^{\prime}(\boldsymbol{r}, t)$ and the cut-off energy $\epsilon_{\infty}^{\prime}(\boldsymbol{r}, t)$. In SW1 it is discussed how a number of hydrodynamical quantities, such as the particle energy $\epsilon^{\prime}$ are calculated by the code MPI-AMRVAC. By adding three additional transport equations to this system, it is possible to also calculate the other three quantities $n^{\prime}(\boldsymbol{r}, t), n_{\mathrm{e} 0}^{\prime}(\boldsymbol{r}, t)$ and $\epsilon_{\infty}^{\prime}(\boldsymbol{r}, t)$. These additional transport equations, in conservative form, are:

$\nabla_{\mu}\left(n^{\prime} U^{\mu}\right)=0$,

$\nabla_{\mu}\left(n^{\prime} n_{0}^{\prime} U^{\mu}\right)=0$,

which is equivalent with $U^{\mu} \nabla_{\mu} n_{0}^{\prime}=0$ because of Eqn. A33, and

$\nabla_{\mu}\left[\epsilon_{\infty}^{\prime}\left(n^{\prime}\right)^{2 / 3} U^{\mu}\right]=-\tilde{\beta}_{\text {sy }}\left(B^{\prime}\right)^{2}\left(\epsilon_{\infty}^{\prime}\right)^{2}\left(n^{\prime}\right)^{2 / 3}$,

with $\tilde{\beta}_{\text {sy }}=2 / 3 \beta_{\text {sy }}$. For more details see for example Camus et al. (2009) or Camus (2009), Eq. 4.102 - 4.103.

Finally, the synchrotron frequency $v^{\prime}$ of a particle with energy $\epsilon^{\prime}$, which moves along a magnetic field $B^{\prime}$ scales as:

$v^{\prime} \propto B_{\perp}^{\prime}\left(\epsilon^{\prime}\right)^{2}$,

Using this result, we end up with the synchrotron frequency dependent version of the particle distribution:

$\mathcal{N} \propto \mathcal{N}_{0}\left(\frac{n_{\mathrm{e}}^{\prime}}{n_{\mathrm{e} 0}^{\prime}}\right)^{\frac{s+2}{3}}\left(1-\sqrt{\frac{v^{\prime}}{v_{\infty}^{\prime}}}\right)^{s-2}$.

When we compare this particle distribution to that of the $\mathcal{N}$-Tracer model A10, we notice the following: In case of the $\mathcal{N}$-Tracer model, we assume that the population of relativistic particles is directly proportional to the mass-density of the proton gas, $n_{\mathrm{e}}^{\prime}(\boldsymbol{r}, t) \propto \rho^{\prime}(\boldsymbol{r}, t)$ and that this proportionality remains constant. The exact value of this proportionality constant does not play a role in the particle distribution, since this constant is divided out. Therefore, the quantities $\rho^{\prime}(\boldsymbol{r}, t)$ and $\rho_{0}^{\prime}(\boldsymbol{r}, t)$ in A10 can simply be replaced by $n_{\mathrm{e}}^{\prime}(\boldsymbol{r}, t)$ and $n_{\mathrm{e} 0}^{\prime}(\boldsymbol{r}, t)$, as in $\mathrm{A} 37$. Then, it can be easily seen that for frequencies well below the cut-off frequency $v^{\prime}<<v_{\infty}^{\prime}$ this particle distribution, and the particle distribution of the $\mathcal{N}$-Tracer model A10 converge, as they should. However, the $\mathcal{N}$-Tracer model allows us to study the emissivity due to (any combination of) the individual jet components at low energies, whereas the $\mathcal{N}$-Cooling model allows us to study the emissivity at all frequencies below the cut-off frequency for the full jet. 


\section{APPENDIX B: MAGNETIC PRESSURE FROM GAS PRESSURE}

The simulations in this paper are hydrodynamical, employing a one-fluid approximation characterized by a single density $\rho$, pressure $P_{\text {gas }}$ and velocity $\boldsymbol{V} .1^{1}$ The bulk density in a certain grid cell is the sum of all separate (jet + ambient medium) components, which allows us to consider the bulk gas pressure $P_{\text {gas }}$ as the sum of its partial contributions:

$P_{\mathrm{gas}}=P_{\mathrm{am}}+P_{\mathrm{jt}}=\frac{\rho \mathcal{R} T}{\mu}=\frac{\mathcal{R} T}{\mu}\left(\rho_{\mathrm{am}}+\rho_{\mathrm{jt}}\right)$.

Here $\mathcal{R}$ is the gas constant and $\mu$ is the mass of the particles in units of hydrogen mass. The subscript "am" refers to the ambient medium and "jt" refers to jet material. In this approximation, we make use of the assumption that all components within a single grid cell have equal temperature $T \propto P_{\text {gas }} / \rho$. The pressure of the jet material is taken to be the sum of its individual components:

$P_{\mathrm{jt}}=\frac{\mathcal{R} T}{\mu} \rho_{\mathrm{jt}}=\frac{\rho \mathcal{R} T}{\mu} \Sigma_{\mathrm{A}} \theta_{\mathrm{A}}=P_{\mathrm{gas}} \Sigma_{\mathrm{A}} \theta_{\mathrm{A}} \equiv \Sigma_{\mathrm{A}} P_{\mathrm{A}}$,

where we used the fact that $\rho_{\mathrm{jt}}=\rho \Sigma_{A} \theta_{A}=\rho \theta_{\mathrm{jt}}$, with $\theta_{\mathrm{jt}}$ being the sum of all jet component mass fractions within a certain grid cell. Under these assumptions one finds that in the one-fluid approximation, the contribution of jet component $A$ to the total pressure in a certain grid cell is equal to $P_{\mathrm{A}}(\boldsymbol{r}, t)=\theta_{\mathrm{A}}(\boldsymbol{r}, t) P_{\text {gas }}(\boldsymbol{r}, t)$.

Next we consider the time evolution of the gas pressure. For an ultra-relativistic gas, the result is already given by Eqn. A14. In general, the gas pressure satisfies an equation of state $P \propto \rho^{\Gamma}$ and satisfies

$\frac{\mathrm{d} P}{\mathrm{~d} \tau}=-\Gamma P(\nabla \cdot U)$,

where $\Gamma$ is the adiabatic (or polytropic) index of the (one-fluid approximation) gas. For a non-relativistic cold gas $\Gamma=5 / 3$, and for an ultra-relativistic gas $\Gamma=4 / 3$. For a gas that is in an intermediate phase, an effective polytropic index $\Gamma_{\text {eff }}(P, \rho)$ can be defined. In our simulations we make use of the Mathews approximation of the Synge equation of state (see for example Synge 1957, Blumenthal \& Mathews 1976 or Goedbloed, Keppens \& Poedts 2019). In this approximation, the adiabatic index is dependent on the particle energy and varies between $4 / 3<\Gamma_{\text {eff }}<5 / 3$ (see SW1 for more details).

Now consider a magnetic field $\boldsymbol{B}=\boldsymbol{B}(\boldsymbol{r}, t)$, with field strength $B \equiv|\boldsymbol{B}|$, and unit vector $\hat{\boldsymbol{b}}=\boldsymbol{B} / B$ along the magnetic field. In the case of ideal MHD, the time evolution of the magnetic pressure $P_{\mathrm{M}}=B^{2} / 8 \pi$ for a regular flow with relativistic velocity field $\boldsymbol{U}(\boldsymbol{r}, t)=\gamma(\boldsymbol{r}, t) \boldsymbol{V}(\boldsymbol{r}, t)$ can be written as (which can easily be seen from Achterberg \& Norman 2018, Eqn. 6.71):

$\frac{\mathrm{d} P_{\mathrm{M}}}{\mathrm{d} \tau}=\gamma \frac{\mathrm{d} P_{\mathrm{M}}}{\mathrm{d} t}=2(\gamma+1) P_{\mathrm{M}}\left[\hat{\boldsymbol{b}} \hat{\boldsymbol{b}}: \tilde{\boldsymbol{\nabla}}\left(\frac{\boldsymbol{U}}{\gamma+1}\right)-\tilde{\boldsymbol{\nabla}} \cdot\left(\frac{\boldsymbol{U}}{\gamma+1}\right)\right]$

The double contraction in this relation is

$\hat{\boldsymbol{b}} \hat{\boldsymbol{b}}: \tilde{\boldsymbol{\nabla}}\left(\frac{\boldsymbol{U}}{\gamma+1}\right) \equiv\left[(\hat{\boldsymbol{b}} \cdot \tilde{\boldsymbol{\nabla}})\left(\frac{\boldsymbol{U}}{\gamma+1}\right)\right] \cdot \hat{\boldsymbol{b}}$,

The operator $\tilde{\boldsymbol{\nabla}}$ stands for the fluid rest-frame gradient operator, expressed via the Lorentz transformation between the fluid rest frame

\footnotetext{
1 In this section we refrain from writing the apostrophe for quantities measured in the fluid rest frame. This applies to the pressure $P$, the magnetic field $\boldsymbol{B}$ (and related quantities) as well as the proper density $\rho$.
}

and oberver's frame in the observer-frame time derivative and spatial derivatives:

$\tilde{\boldsymbol{\nabla}} \equiv \boldsymbol{\nabla}+\boldsymbol{U}\left(\frac{\partial}{\partial t}+\frac{(\boldsymbol{U} \cdot \boldsymbol{\nabla})}{\gamma+1}\right)$.

In general, $\mathrm{B} 3$ and $\mathrm{B} 4 \mathrm{3}$ do not have the same form, and a simple relationship between the gas- and magnetic pressure is not available. However, if the field is isotropically turbulent (entangled) on small scales, the following relation holds:

$<b_{i} b_{j}>=\frac{\delta_{i j}}{3}$,

Here the average $\langle\cdots\rangle$ is a spatial average over a small volume with a linear size much smaller than the size of the system. In this case one has

$\left\langle\hat{\boldsymbol{b}} \hat{\boldsymbol{b}}: \tilde{\boldsymbol{\nabla}}\left(\frac{\boldsymbol{U}}{\gamma+1}\right)\right\rangle \simeq \frac{1}{3} \tilde{\boldsymbol{\nabla}} \cdot\left(\frac{\boldsymbol{U}}{\gamma+1}\right)$.

With this approximation the time evolution of the magnetic pressure collapses to

$\frac{\mathrm{d} P_{\mathrm{M}}}{\mathrm{d} \tau}=-\frac{4}{3} P_{\mathrm{M}}\left[(\gamma+1) \tilde{\nabla} \cdot\left(\frac{\boldsymbol{U}}{\gamma+1}\right)\right]=-\frac{4}{3} P_{\mathrm{M}}(\nabla \cdot U)$,

Here we use the relation $(\gamma+1) \tilde{\boldsymbol{\nabla}} \cdot[\boldsymbol{U} /(\gamma+1)]=\partial \gamma / \partial t+\boldsymbol{\nabla} \cdot \boldsymbol{U}=$ $\nabla \cdot U$. Comparing this with relation $(\mathrm{B} 3)$ one sees that the magnetic pressure behaves as an ultra-relativistic gas with polytropic index $\Gamma_{\mathrm{M}}=4 / 3$. One concludes that the magnetic pressure scales as

$P_{\mathrm{M}}^{\prime} \propto\left(\rho^{\prime}\right)^{4 / 3} \propto\left(n_{\mathrm{e}}^{\prime}\right)^{4 / 3}$,

where we re-instated the apostrophe notation for quantities in the fluid rest frame. The ratio of the gas pressure to the magnetic pressure (the so-called plasma beta) scales as

$\frac{P_{\text {gas }}^{\prime}}{P_{\mathrm{M}}^{\prime}} \equiv \beta_{\mathrm{p}} \propto\left(\rho^{\prime}\right)^{\Gamma-4 / 3}$.

When the gas is relativistically hot $(\Gamma=4 / 3)$, the plasma beta becomes a constant. The energy of the system will be minimized in the case where $\beta_{\mathrm{p}}$ is close to unity. In that case there is equipartition between the gas pressure and the pressure stored in the magnetic field. This assumption is often made when trying to get an estimate on the magnetic field strength. However, in our simulations the plasma beta will still be weakly dependent on the energy density of the gas through the effective polytropic index $\Gamma$ of the gas. Finally, we make the assumption that there is no magnetic field present in the ambient intergalactic medium. In that case, the magnetic pressure and magnetic field strength $B^{\prime}$ in the simulations will be completely determined by the jet gas pressure according to:

$P_{\mathrm{M}}^{\prime}=\frac{\left(B^{\prime}\right)^{2}}{8 \pi} \propto P_{\mathrm{jt}}^{\prime}\left(\rho^{\prime}\right)^{4 / 3-\Gamma_{\mathrm{eff}}}=\left[P_{\mathrm{gas}}^{\prime} \Sigma_{\mathrm{A}} \theta_{A}^{\prime}\right]\left(\rho^{\prime}\right)^{4 / 3-\Gamma_{\mathrm{eff}}}$.

The quantities $P_{\text {gas }}^{\prime}, \theta_{\mathrm{A}}^{\prime}, \rho^{\prime}$ are all calculated by the code, and the effective polytropic index $\Gamma_{\text {eff }}$ is determined at any point in time and space during the simulation. Then using the assumption of an entangled field will allow us to get a detailed estimate of the magnetic pressure at any point and time in the flow.

\section{APPENDIX C: MAGNETIC FIELD CONFIGURATION AND PROJECTION EFFECTS}

As shown in the previous section, we can approximate the magnetic pressure for an isotropically entangled magnetic field through the gas pressure, tracer values, mass density and adiabatic index 
of the jet components. In the remainder of this section, we expand our synchrotron emission model further by taking into account the effect of large-scale (ordered) magnetic fields within the jets. With the ordered fields, projection effects come into play, as shown in the following sections.

\section{C1 Perpendicular magnetic field component}

As mentioned earlier, the magnitude of the synchrotron emissivity depends on $B_{\perp}^{\prime}$, the absolute value of the magnetic field component perpendicular to the particle's velocity in the plasma rest-frame. Therefore, the contribution to the synchrotron emission from relativistic particles spiralling around a magnetic field $\boldsymbol{B}^{\prime}$ in the plasma rest-frame, in a direction $\boldsymbol{n}^{\prime}$ (the unit vector along the line of sight, as measured in the plasma rest-frame) will be determined by the cross-product of $\boldsymbol{B}^{\prime}$ and $\boldsymbol{n}^{\prime}$, in other words:

$B_{\perp}^{\prime}=\left|B^{\prime} \times n^{\prime}\right|=\left|B^{\prime}\right| \sqrt{1-\left(\boldsymbol{b}^{\prime} \cdot \boldsymbol{n}^{\prime}\right)^{2}}$,

where $\boldsymbol{b}^{\prime}$ is the unit vector pointing in the direction of $\boldsymbol{B}^{\prime}$ and $B^{\prime}=\left|\boldsymbol{B}^{\prime}\right|=\sqrt{\boldsymbol{B}^{\prime} \cdot \boldsymbol{B}^{\prime}}$. Now consider a photon that is emitted in a direction $\boldsymbol{n}^{\prime}$ in the plasma rest-frame. When this photon reaches the observer, it appears to have been emitted from a different direction $\boldsymbol{n}$, an effect caused by general Lorentz transformations on vectors. It can be shown that the relation between $\boldsymbol{n}^{\prime}$ and $\boldsymbol{n}$ is given by (see for example Camus 2009 Eq. 4.37):

$\boldsymbol{n}^{\prime}=\mathcal{D}\left[\boldsymbol{n}+\left(\frac{\boldsymbol{n} \cdot \boldsymbol{\beta}}{V^{2}}(\gamma-1)-\gamma\right) \boldsymbol{\beta}\right]$

and this can be rewritten as:

$\boldsymbol{n}^{\prime}=\mathcal{D} \boldsymbol{n}-(\mathcal{D}+1) \frac{\gamma}{\gamma+1} \boldsymbol{\beta}$,

with as before $\beta$ the bulk 3-velocity of the plasma in units of $c$; $\gamma$ the Lorentz factor and $\mathcal{D}$ the Doppler factor. So in a HD simulation, by assuming a certain magnetic field configuration in the plasma rest-frame $\boldsymbol{B}^{\prime}$ and choosing a line of sight $\boldsymbol{n}$, one can always calculate the contribution to the synchrotron emissivity from the perpendicular magnetic field component $B_{\perp}^{\prime}$ by combining (C1) and $(\mathrm{C} 3$.

In this paper we use 2.5D simulations, where the jets are injected along the $Z$-direction and the jets are axisymmetrical in the azimuthal $(\phi$-)direction. Since the simulations are axisymmetrical, the line of sight can simply be chosen by one free parameter: the viewing angle $\vartheta=\angle\left(\hat{\boldsymbol{e}}_{Z}, \boldsymbol{n}\right)$. The line of sight vector can in general be written as:

$\boldsymbol{n}=\left(\sin (\vartheta) \cos \left(\varphi_{0}\right), \sin (\vartheta) \sin \left(\varphi_{0}\right), \cos (\vartheta)\right)$.

The choice for $\varphi_{0}$ in this case, however, is arbitrary and we take this to be $\varphi_{0}=0$. Then the line of sight vector simplifies to:

$\boldsymbol{n}=(\sin (\vartheta), 0, \cos (\vartheta))$.

In the following sections we will discuss the magnetic field configurations that we consider for our synthesized synchrotron maps. Since we are focussing on intensity variation maps, the exact scale is arbitrary. This allows us to set the imposed magnetic field in the plasma rest-frame $B^{\prime}$ to order unity at the jet inlet.

\section{C2 Isotropically entangled field configuration}

A very entangled magnetic field, on a larger scale does not have a preferable direction. That means that the magnitude of the perpendicular magnetic field component $B_{\perp}^{\prime}$ is not dependent on the viewing angle $\vartheta$, so there are no projection effects. We find for the perpendicular component of the entangled field:

$\left(b_{\perp, \text { ent }}^{\prime}\right)^{2} \propto\left|\boldsymbol{b}_{\text {ent }}^{\prime} \times \boldsymbol{n}^{\prime}\right|^{2}=\left(b_{\text {ent }}^{\prime}\right)^{2}\left(1-\left(\boldsymbol{n}^{\prime} \cdot \boldsymbol{b}_{\text {ent }}^{\prime}\right)^{2}\right)=1-\left(\boldsymbol{n}^{\prime} \cdot \boldsymbol{b}_{\text {ent }}^{\prime}\right)^{2}$.(C6)

Since the field is isotropically entangled, equation B7 applies:

$\left\langle\left(\boldsymbol{n}^{\prime} \cdot \boldsymbol{b}_{\mathrm{ent}}^{\prime}\right)^{2}\right\rangle=\left(n^{\prime}\right)^{i}\left(n^{\prime}\right)^{j}\left\langle b_{i}^{\prime} b_{j}^{\prime}\right\rangle=\frac{1}{3}$,

so that averaging the magnetic field squared on a large enough volume one finds:

$\left\langle\left(b_{\perp, \text { ent }}^{\prime}\right)^{2}\right\rangle=1-\left\langle\boldsymbol{n}^{\prime} \cdot \boldsymbol{b}_{\text {ent }}^{\prime}\right\rangle^{2}=\frac{2}{3}$.

Making use of our earlier result for the magnetic pressure B12, we find for the magnitude of the perpendicular field component of the entangled magnetic field:

$\left(B_{\perp, \text { ent }}^{\prime}\right)^{2} \propto \frac{2}{3} P_{\text {gas }}^{\prime}\left(\rho^{\prime}\right)^{\frac{4-3 \Gamma}{3}} \Sigma_{A} \theta_{\mathrm{A}}^{\prime}$.

When the magnetic field configuration is assumed to be purely (isotropically) entangled, the factor $2 / 3$ can be dropped immediately, since we are merely studying the intensity variations of the synchrotron emission. However, when apart from the entangled field, additional ordered field configurations are assumed, the factor $2 / 3$ needs to be included.

\section{C3 Ordered field configurations}

In this section we discuss a method to obtain ordered magnetic fields in the rest frame of the plasma, to calculate their contributions to the synchrotron emission. The jet is most conveniently described using a cylindrical coordinate system $(R, \phi, Z)$, where the central engine is at rest (the central engine rest-frame) and the jet propagates outward along the $Z$-axis. In this reference frame the magnetic field at some point $\boldsymbol{r}$ has cylindrical field components $\left(B_{R}, B_{\phi}, B_{Z}\right)$. When the magnetic field configuration of a jet has a helical structure, the radial field component $B_{R}$ is zero for a constant jet radius. In that case, the pitch angle $\kappa$ between the azimuthal field component $B_{\phi}$ and the poloidal field component $B_{Z}$ can be defined as:

$\tan (\kappa)=\frac{B_{Z}}{B_{\phi}}$,

where $\kappa=0^{\circ}$ corresponds to a pure azimuthal field and $\kappa=90^{\circ}$ corresponds to a pure poloidal field. In Cartesian coordinates, for a jet with a helical magnetic field structure we find:

$\boldsymbol{B}=\left(\begin{array}{c}-B_{\phi} \sin (\phi) \cos (\kappa) \\ B_{\phi} \cos (\phi) \cos (\kappa) \\ B_{Z} \sin (\kappa)\end{array}\right)$.

For a pure poloidal field we write $\boldsymbol{B}_{\mathrm{pol}}=B_{\mathrm{pol}} \boldsymbol{b}_{\mathrm{pol}}$, with

$\boldsymbol{b}_{\mathrm{pol}}=\left(\begin{array}{c}0 \\ 0 \\ 1\end{array}\right)$.

The magnitude of the poloidal field, $B_{\text {pol }}$, is easily determined: the conservation of poloidal magnetic flux can be written as:

$\pi R_{\mathrm{jt}}^{2} B_{\mathrm{pol}}^{\prime}(R)=\Phi=$ constant,

where $R_{\mathrm{jt}}(Z)$ is the jet radius at a distance $Z$ from the jet inlet. From this it follows that the magnitude of the poloidal field scales as:

$B_{\mathrm{pol}}^{\prime}(R) \propto \frac{1}{R_{\mathrm{jt}}^{2}}$ 
For a pure azimuthal field we write $\boldsymbol{B}_{\mathrm{azm}}=\mathrm{B}_{\mathrm{azm}} \boldsymbol{b}_{\mathrm{azm}}$, with

$\boldsymbol{b}_{\mathrm{azm}}=\left(\begin{array}{c}-\sin (\phi) \\ \cos (\phi) \\ 0\end{array}\right)$.

Also for this magnetic field configuration, the magnitude $B_{\text {azm }}$ is easily determined: Ampère's law determines the azimuthal field in terms of the current as:

$B_{\mathrm{azm}}(R)=\frac{2 I(<R)}{c R}$,

where $I(<R)$ is the current that passes through a disk surface with radius $R$, centred around the jet axis, and $c$ is the speed of light. For a homogeneous and structureless jet, a simple assumption can be made that the electrical current is uniformly distributed over the jet cross section so that the current density $j$ equals:

$j=\frac{I_{\mathrm{jt}}}{\pi R_{\mathrm{jt}}^{2}}=$ constant,

where $I_{\mathrm{jt}}$ is the total electrical current that passes through the jet at any given point along the jet axis. In that case the electrical current is proportional to $I(<R) \propto j R^{2}$, so that:

$B_{\mathrm{azm}}(R) \propto \frac{R}{R_{\mathrm{jt}}^{2}}$.

The synchrotron emissivity in a certain direction depends on the field component perpendicular to the ray direction, all in the local plasma rest frame. Therefore, we will first transform the magnetic fields back to the plasma rest-frame, and after we will calculate the field component perpendicular to the line of sight.

Magnetic fields transform under Lorentz transformations according to:

$\boldsymbol{B}^{\prime}=\frac{1}{\gamma}\left(\boldsymbol{B}+\frac{\gamma^{2}}{\gamma+1} \boldsymbol{\beta}(\boldsymbol{\beta} \cdot \boldsymbol{B})\right)$

with the meaning $\beta$ and $\gamma$ as before. Now that we have the magnetic field in the plasma rest-frame $(\mathrm{C} 19)$, as well as the line of sight vector in the plasma rest-frame (C3), both in Cartesian coordinates, the magnetic field component perpendicular to the line of sight is easily obtained:

$\boldsymbol{B}_{\perp, \text { ord }}^{\prime}=\boldsymbol{B}_{\text {ord }}^{\prime} \times \boldsymbol{n}^{\prime}$,

where subscript "ord" stands for ordered, which can be purely azimuthal, purely poloidal, or helical. From this field component, we can calculate the magnetic energy, which is proportional to:

$\left(B_{\perp, \text { ord }}^{\prime}\right)^{2}=\left|\boldsymbol{B}_{\text {ord }}^{\prime} \times \boldsymbol{n}^{\prime}\right|^{2}=\left(B_{\text {ord }}^{\prime}\right)^{2}\left(1-\left(\boldsymbol{n}^{\prime} \cdot \boldsymbol{b}_{\text {ord }}^{\prime}\right)^{2}\right)$.

\section{C4 Mixed field configuration}

When two components (such as a jet with a spine-sheath structure) start out with an ordered magnetic field configuration and these components are advected, turbulence and hydrodynamical instabilities will lead to mixing between these two components. A jet for example is thought to have a helical magnetic field structure near the central engine of a AGN. As the jet propagates through the ambient medium, instabilities and turbulence will lead to a larger fraction of entanglement. Therefore, it is to be expected that astrophysical jets and their surrounding cocoon will consist of a mix of large-scale (ordered) fields and entangled (random) fields. There is another simple argument that predicts the existence of large-scale, ordered fields: if there are large-scale electrical currents, such as a current along the jet's axis, they will generate a large-scale ordered azimuthal field. So even though turbulence is present, if large-scale currents are not disrupted the large-scale field remains. In the case where magnetic fields do not play a dynamically important role, one can make a number of simple assumptions that could describe this evolution based on pure hydrodynamic simulations.

Consider a system that has an isotropically entangled magnetic field $\boldsymbol{B}_{\text {ent }}^{\prime}$ component, as well as an ordered magnetic field component $\boldsymbol{B}_{\text {ord }}^{\prime}$ which can be a poloidal field, an azimuthal field or a helical field. Then the total magnetic field can be written as:

$\boldsymbol{B}^{\prime}=\boldsymbol{B}_{\text {ent }}^{\prime}+\boldsymbol{B}_{\text {ord }}^{\prime}$.

Since the average over a large enough subvolume yields $\left\langle\boldsymbol{B}_{\text {ent }}^{\prime}\right\rangle=0$, the average of the total magnetic pressure is proportional to:

$\left(B^{\prime}\right)^{2} \equiv\left\langle\left(B^{\prime}\right)^{2}\right\rangle=\left(B_{\text {ord }}^{\prime}\right)^{2}+\left(B_{\text {ent }}^{\prime}\right)^{2}$,

where $\left(B_{\text {ent }}^{\prime}\right)^{2}=\left\langle\left|\boldsymbol{B}_{\text {ent }}^{\prime}\right|^{2}\right\rangle$ is implied. We can write the magnetic pressure of the field components as a fraction of the total magnetic pressure in the following way:

$\Lambda=\frac{\left(B_{\text {ent }}^{\prime}\right)^{2}}{\left(B_{\text {ent }}^{\prime}\right)^{2}+\left(B_{\text {ord }}^{\prime}\right)^{2}}$,

and

$1-\Lambda=\frac{\left(B_{\text {ord }}^{\prime}\right)^{2}}{\left(B_{\text {ent }}^{\prime}\right)^{2}+\left(B_{\text {ord }}^{\prime}\right)^{2}}$.

For the average contribution from the perpendicular magnetic pressure we find:

$\left\langle\left(B_{\perp}^{\prime}\right)^{2}\right\rangle=\left(B_{\perp, \text { ord }}^{\prime}\right)^{2}+\frac{2}{3}\left(B_{\text {ent }}^{\prime}\right)^{2}$,

from which it easily follows that:

$\left(B_{\perp}^{\prime}\right)^{2}=\left(B^{\prime}\right)^{2}\left\{\frac{2}{3} \Lambda+(1-\Lambda)\left|\boldsymbol{b}_{\text {ord }}^{\prime} \times \boldsymbol{n}^{\prime}\right|^{2}\right\}$,

or equivalently

$\left(B_{\perp}^{\prime}\right)^{2}=\left(B^{\prime}\right)^{2}\left\{\frac{2}{3} \Lambda+(1-\Lambda)\left(1-\left(\boldsymbol{n}^{\prime} \cdot \boldsymbol{b}_{\text {ord }}^{\prime}\right)^{2}\right)\right\}$,

where the average over a large enough subvolume is implied.

This is still a general result. Now if we were to make the assumption that not just the entangled magnetic field pressure $\left(B_{\text {ent }}^{\prime}\right)^{2}$, but the total magnetic pressure $\left(B^{\prime}\right)^{2}$ scales with the gas pressure as in (C9), the magnetic pressure from the mixed field configuration C28 can be calculated. Since $\Lambda$ is the fraction of magnetic pressure from the entangled field compared to that of the total magnetic field, $\Lambda=1$ corresponds to $\left(B_{\text {ord }}^{\prime}\right)^{2}=0$; while $\Lambda=0$ corresponds to $\left(B_{\text {ent }}^{\prime}\right)^{2}=0$. For $\Lambda=0.5$ the magnetic pressure is equally distributed between the ordered field and the entangled field. 